\title{
Article \\ Satellitome Analysis of the Pacific Oyster Crassostrea gigas Reveals New Pattern of Satellite DNA Organization, Highly Scattered across the Genome
}

\author{
Monika Tunjić-Cvitanić ${ }^{1}$, Juan J. Pasantes ${ }^{2}$ D , Daniel García-Souto ${ }^{3,4}$ (D), Tonči Cvitanić ${ }^{5}$, Miroslav Plohl ${ }^{1}$ \\ and Eva Šatović-Vukšić $1, * \mathbb{D}$
}

1 Division of Molecular Biology, Ruđer Bošković Institute, 10000 Zagreb, Croatia; mtunjic@irb.hr (M.T.-C.); plohl@irb.hr (M.P.)

2 Centro de Investigación Mariña, Universidade de Vigo, Dpto de Bioquímica, Xenética e Inmunoloxía, 36310 Vigo, Spain; pasantes@uvigo.es

3 Genomes and Disease, Centre for Research in Molecular Medicine and Chronic Diseases (CIMUS), Universidade de Santiago de Compostela, 15706 Santiago de Compostela, Spain; danielgarciasouto@gmail.com

4 Department of Zoology, Genetics and Physical Anthropology, Universidade de Santiago de Compostela, 15706 Santiago de Compostela, Spain

5 Rimac Automobili d.o.o., Ljubljanska ulica 7, 10431 Sveta Nedelja, Croatia; tonci.cvitanic@gmail.com

* Correspondence: esatovic@irb.hr

\section{check for}

updates

Citation: Tunjić-Cvitanić, M.; Pasantes, J.J.; García-Souto, D.;

Cvitanić, T.; Plohl, M.; Šatović-Vukšić,

E. Satellitome Analysis of the Pacific

Oyster Crassostrea gigas Reveals New

Pattern of Satellite DNA

Organization, Highly Scattered across the Genome. Int. J. Mol. Sci. 2021, 22, 6798. https://doi.org/10.3390/ ijms 22136798

Academic Editor: Naoyuki Kataoka

Received: 9 June 2021

Accepted: 19 June 2021

Published: 24 June 2021

Publisher's Note: MDPI stays neutral with regard to jurisdictional claims in published maps and institutional affiliations.

Copyright: (c) 2021 by the authors. Licensee MDPI, Basel, Switzerland. This article is an open access article distributed under the terms and conditions of the Creative Commons Attribution (CC BY) license (https:// creativecommons.org/licenses/by/ $4.0 /)$.

\begin{abstract}
Several features already qualified the invasive bivalve species Crassostrea gigas as a valuable non-standard model organism in genome research. C. gigas is characterized by the low contribution of satellite DNAs (satDNAs) vs. mobile elements and has an extremely low amount of heterochromatin, predominantly built of DNA transposons. In this work, we have identified 52 satDNAs composing the satellitome of $C$. gigas and constituting about $6.33 \%$ of the genome. Satellitome analysis reveals unusual, highly scattered organization of relatively short satDNA arrays across the whole genome. However, peculiar chromosomal distribution and densities are specific for each satDNA. The inspection of the organizational forms of the 11 most abundant satDNAs shows association with constitutive parts of Helitron mobile elements. Nine of the inspected satDNAs are dominantly found in mobile element-associated form, two mostly appear standalone, and only one is present exclusively as Helitron-associated sequence. The Helitron-related satDNAs appear in more chromosomes than other satDNAs, indicating that these mobile elements could be leading satDNA propagation in C. gigas. No significant accumulation of satDNAs on certain chromosomal positions was detected in C. gigas, thus establishing a novel pattern of satDNA organization on the genome level.
\end{abstract}

Keywords: satellite DNA; satellitome; mobile element; Helitron; bivalve; Crassostrea gigas

\section{Introduction}

Large fractions of eukaryotic genomes are composed of repetitive DNA sequences that could be either repeated in tandem, among which satellite DNAs (satDNAs) dominate, or are interspersed, due to the activity of mobile elements [1-5]. Nowadays, comprehensive analyses of repetitive DNA in eukaryotic genomes have been enabled by the employment of NGS (Next Generation Sequencing) methodologies, complemented with specialized bioinformatic tools and programs [6,7]. NGS-related bioinformatics allowed revealing either the complete set of repetitive DNA sequences, the repeatome [8], or the broad collection of satDNAs, the satellitome [9], present in eukaryotic genomes. These approaches showed surprisingly large numbers of satDNAs in eukaryotic genomes, i.e., 62 in the migratory locust Locusta migratoria [9], 129 in the Australian morabine grasshoppers of the genus Vandiemenella [10], 164 in the characiform fish Megaleporinus microcephalus [11] and 37 in the plant Passiflora organensis [12]. Bioinformatic approaches combined with fluorescence 
in situ hybridization (FISH) yielded new information about the structure, the chromosomal location, and the evolution of these sequences within or among genomes [13-16].

From the generally accepted point of view, classical satDNAs are organized in long arrays, consisting of hundreds to thousands of monomers repeated in tandem, and occupying the constitutive heterochromatin. Heterochromatin is mostly located at pericentromeric and subtelomeric chromosomal domains, being sometimes also found at interstitial loci of the chromosomal arms [4]. However, satDNA sequences were also detected outside of the heterochromatin, in different organizational forms. In some cases, the same sequence can simultaneously appear in the genome in the form of a classical satDNA, and in the form of short arrays, or as single monomers and monomer fragments located in euchromatic genome compartments [17-21]. Some short arrays are at the same time structural components of the central parts of mobile elements [17,22-24]. Due to this diversity, the study of the biology of satDNA sequences requires a versatile pool of model systems.

Bivalve mollusks hold great economic and ecological importance. Their commercial significance is unquestionable in aquaculture, where they have several million-ton productions per year due to their high nutritional value [25]. The ecological impact of these organisms is emphasized when invasive bivalve species start to occupy new environments, significantly affecting native organisms in the new habitat [26]. The research interest encompassing all aspects of bivalve biology is fast-growing [27,28], and is accompanied by an increasing number of sequenced genomes (31 until March 2021, NCBI) forwarding the bivalves rapidly towards well-established model organisms [29].

The estimated content of repetitive DNA in the majority of bivalve genomes sequenced so far is high, about $35 \%$, while the contribution of the satDNA fraction is low, less than $2 \%$ of the genomic DNA (i.e., [30-33]). Although satDNA content is regularly underestimated in sequenced genomes, the results of classical restriction enzyme digestion and cloning are roughly in agreement with this estimation. The 26 different satDNAs from 48 bivalve species experimentally detected so far showed low genomic contents, some of them significantly less than $1 \%$ of the genomic DNA (reviewed in [34]). Altogether, this indicates that the presence of numerous, low copy satDNAs together with abundant mobile elements could be a specificity of bivalve genomes.

As the unambiguous classification of repetitive sequences in bivalve genomes is often difficult, many of them, about 70\%, remained unassigned in reported cases [30]. For instance, although in C. gigas, the first sequenced bivalve genome, $36 \%$ of the assembled sequences were identified as repetitive, $62 \%$ of them could not be assigned to any of the known categories. Among those assigned, the majority were characterized as mobile elements, while satDNAs were estimated to make only $1.2 \%$ of the genome [35]. Nevertheless, tandem repeats belonging to the most abundant HindIII/Cg170 satDNA, were experimentally estimated to build $1-4 \%$ of the genome [36]. Short arrays of satDNA belonging to this sequence, in average about six monomers long, were regularly found in central parts of mobile elements belonging to the family of Helitrons/Helentrons [19,37], known to employ rolling circle mechanisms in their spreading process [38].

In accordance, the newly assembled $C$. gigas representative genome has a remarkably high number of predicted Helitron-related sequences when compared to several other molluscan genome assemblies [39]. Such a hybrid structure of mobile elements holding tandem repeats could explain the difficulties in both categorizing repetitive sequences in bivalves and determining the precise contribution of each type to the repeatome. For example, after the RepeatExplorer analysis, tandem repeats from central parts of sequences (later recognized as Helitrons) were placed into one cluster and classified as a satellite DNA, while sequences surrounding these central repeats were assigned to other, nonclassified, clusters [35]. In that respect, only more detailed analysis of such sequences could resolve whether they appear in the form of the classical long-array satDNAs, shortarray satDNAs, long-array satDNAs that are associated with mobile elements, short-array satDNAs associated with mobile elements, or in all of the abovementioned forms. 
Despite extensive satellitome and repeatome studies in many species, little is known about how repetitive DNA sequences are structured in bivalves and thus need to be explored in detail at the whole-genome level. The aforementioned characteristics qualify C. gigas as a valuable non-standard model species in exploring both the satellitome and the organizational patterns of repetitive DNA sequences. In this study, the satellitome of a bivalve species, the Pacific oyster C. gigas, was analyzed, for the first time in our knowledge, using bioinformatic approaches accompanied by in silico and in situ chromosomal localization of the most prominent sequences repeated in tandem. Furthermore, in order to better understand the evolutionary processes structuring $C$. gigas genome, we disclosed the preferential organizational forms of the most abundant tandem repeats in this species.

\section{Results}

\subsection{SatDNA Content of the C. gigas Genome}

In order to obtain a comprehensive overview of the satDNAs in the Pacific oyster genome, several rounds of RepeatExplorer2 clustering were performed on four randomly subsampled sets of pair-end NGS reads. The combined results of the four analyses (Table S1) resulted in a pool of 52 sequences repeated in tandem that was considered as the satellitome of this species. The detected satDNAs exhibit a broad range of monomer lengths, varying from 21 (CgiSat43) to 3287 bp (CgiSat38), and AT contents (from 44 to 76.1\%; Table 1). SatDNAs with 160-180 bp monomer lengths dominate (Figure 1), constituting 33\% of the satellitome.

The abundance of satDNAs comprising the satellitome (averaged from the outputs of the four analyses) is relatively low, ranging from $0.01 \%$ (which is the bottom cut-off level of the program output) to $0.72 \%$ of the genome (Table 1 ).

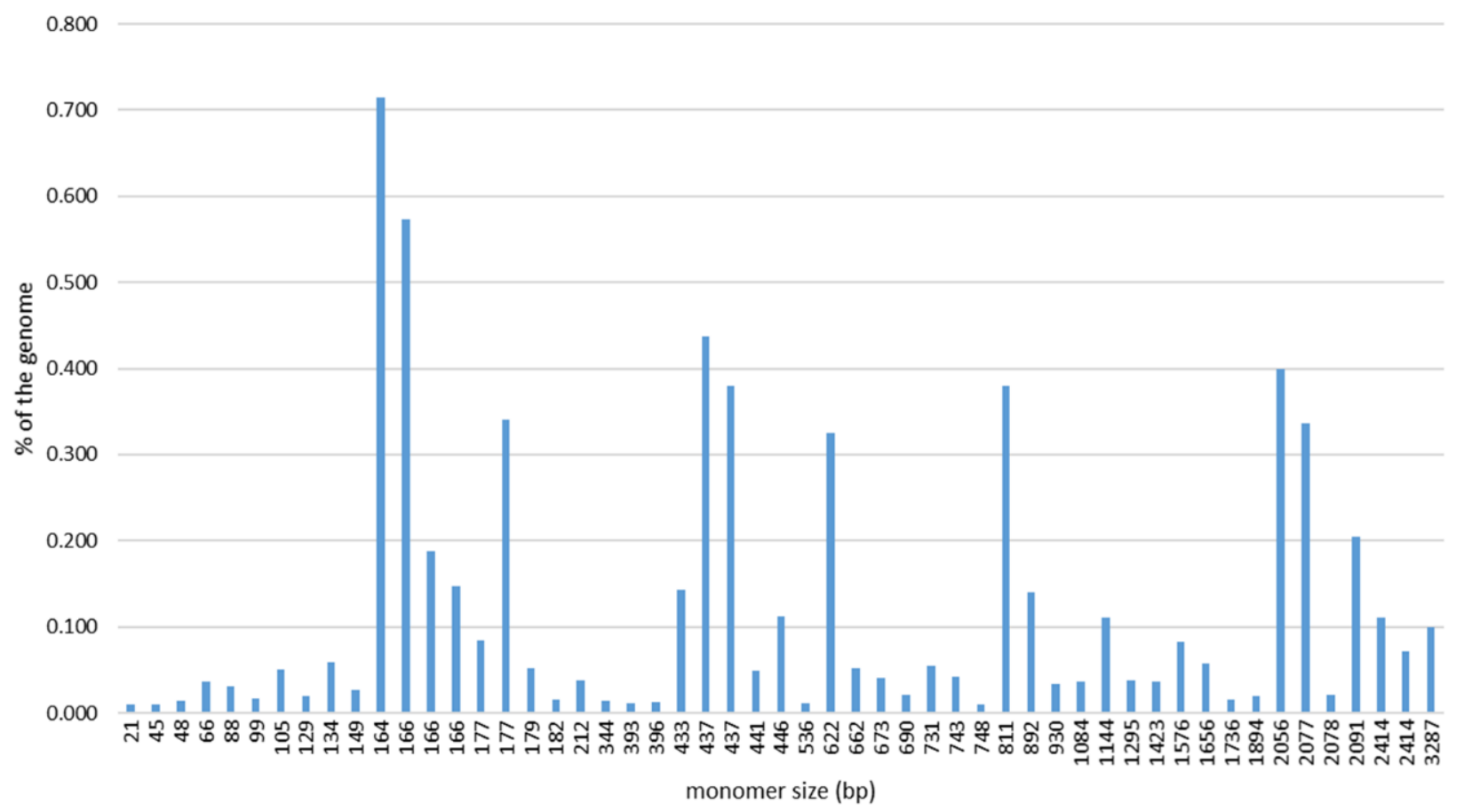

Figure 1. Monomer sizes (bp) and genomic contributions (\%) of the 52 satDNAs of C. gigas including 2 variants of CgiSat01. 
Table 1. Main characteristics of the 52 satDNAs constituting the satellitome of $C$. gigas.

\begin{tabular}{|c|c|c|c|c|c|}
\hline satDNA & $\begin{array}{l}\text { Monomer Length } \\
\text { (bp) }\end{array}$ & $\begin{array}{c}\% \text { of the Genome } \\
\text { (Average) }\end{array}$ & $\begin{array}{c}\% \text { of the } \\
\text { Satellitome }\end{array}$ & $\%$ AT & Repbase \\
\hline CgiSat01a & 164 & 0.72 & 11.29 & 59.8 & DNA/Helitron \\
\hline CgiSat01b & 166 & 0.57 & 9.04 & 57.2 & DNA/Helitron \\
\hline CgiSat02 & 437 & 0.44 & 6.91 & 65.7 & DNA/Helitron \\
\hline CgiSat03 & 437 & 0.38 & 6.00 & 67.5 & DNA/Helitron \\
\hline CgiSat04 & 2077 & 0.34 & 5.32 & 67.3 & DNA/Helitron \\
\hline CgiSat05 & 811 & 0.38 & 6.00 & 67.6 & DNA/Helitron \\
\hline CgiSat06 & 622 & 0.33 & 5.13 & 67.4 & DNA/Helitron \\
\hline CgiSat07 & 2091 & 0.21 & 3.24 & 67.0 & DNA/Helitron \\
\hline CgiSat08 & 166 & 0.19 & 2.96 & 67.5 & DNA/Helitron \\
\hline CgiSat09 & 166 & 0.15 & 2.33 & 63.3 & DNA/Helitron \\
\hline CgiSat10 & 433 & 0.14 & 2.26 & 63.0 & DNA/Helitron \\
\hline CgiSat11 & 892 & 0.14 & 2.21 & 64.6 & LTR/BEL \\
\hline CgiSat12 & 446 & 0.11 & 1.78 & 64.8 & - \\
\hline CgiSat13 & 2414 & 0.11 & 1.74 & 67.4 & DNA/Kolobok \\
\hline CgiSat14 & 1144 & 0.11 & 1.74 & 68.9 & DNA/Helitron \\
\hline CgiSat15 & 177 & 0.08 & 1.32 & 68.9 & - \\
\hline CgiSat16 & 2414 & 0.07 & 1.12 & 66.8 & DNA/Kolobok \\
\hline CgiSat17 & 134 & 0.06 & 0.92 & 76.1 & DNA/Helitron \\
\hline CgiSat18 & 731 & 0.05 & 0.86 & 63.9 & Interspersed Repeat \\
\hline CgiSat19 & 212 & 0.04 & 0.60 & 72.6 & $\begin{array}{lll}1 & -\end{array}$ \\
\hline CgiSat20 & 662 & 0.05 & 0.81 & 65.4 & - \\
\hline CgiSat21 & 441 & 0.05 & 0.77 & 69.2 & DNA/Helitron \\
\hline CgiSat22 & 743 & 0.04 & 0.66 & 53.3 & LTR/Gypsy \\
\hline CgiSat23 & 1295 & 0.04 & 0.60 & 64.9 & DNA/MuDR \\
\hline CgiSat24 & 66 & 0.04 & 0.58 & 57.6 & - \\
\hline CgiSat25 & 88 & 0.03 & 0.49 & 61.4 & NonLTR/R1 \\
\hline CgiSat26 & 1894 & 0.02 & 0.30 & 60.4 & LTR/Gypsy \\
\hline CgiSat27 & 99 & 0.02 & 0.26 & 60.6 & - \\
\hline CgiSat28 & 182 & 0.02 & 0.25 & 44.0 & DNA/MuDR \\
\hline CgiSat29 & 344 & 0.01 & 0.23 & 52.9 & DNA/DNA4-44 \\
\hline CgiSat30 & 48 & 0.01 & 0.22 & 68.7 & - \\
\hline CgiSat31 & 393 & 0.01 & 0.18 & 52.2 & IntegratedVirus/DNAV \\
\hline CgiSat32 & 536 & 0.01 & 0.17 & 66.6 & DNA/Mariner \\
\hline CgiSat33 & 105 & 0.05 & 0.80 & 46.7 & - \\
\hline CgiSat34 & 1423 & 0.04 & 0.57 & 67.3 & DNA/DNA3-8 \\
\hline CgiSat35 & 690 & 0.02 & 0.32 & 68.8 & DNA/IS3EU \\
\hline CgiSat36 & 45 & 0.01 & 0.16 & 60.0 & - \\
\hline CgiSat37 & 177 & 0.34 & 5.37 & 56.5 & DNA/Helitron \\
\hline CgiSat38 & 3287 & 0.10 & 1.58 & 68.0 & DNA/DNA2-7 \\
\hline CgiSat39 & 179 & 0.05 & 0.82 & 52.0 & - \\
\hline CgiSat40 & 673 & 0.04 & 0.65 & 62.9 & DNA/Crypton \\
\hline CgiSat41 & 1736 & 0.02 & 0.24 & 66.5 & DNA/Ginger1 \\
\hline CgiSat42 & 930 & 0.03 & 0.52 & 66.9 & DNA/DNA4-31 \\
\hline CgiSat43 & 21 & 0.01 & 0.16 & 66.7 & - \\
\hline CgiSat44 & 748 & 0.01 & 0.16 & 68.6 & LTR/DIRS \\
\hline CgiSat45 & 1576 & 0.08 & 1.31 & 64.1 & DNA/DNA4-2 \\
\hline CgiSat46 & 149 & 0.03 & 0.41 & 54.4 & - \\
\hline CgiSat47 & 129 & 0.02 & 0.30 & 69.8 & DNA/IS3EU \\
\hline CgiSat48 & 2056 & 0.40 & 6.32 & 64.8 & DNA/Helitron \\
\hline CgiSat49 & 1084 & 0.04 & 0.57 & 69.4 & DNA/DNA2-12 \\
\hline CgiSat50 & 2078 & 0.02 & 0.33 & 64.1 & - \\
\hline CgiSat51 & 396 & 0.01 & 0.19 & 63.1 & LTR/Gypsy \\
\hline CgiSat52 & 1656 & 0.06 & 0.92 & 61.5 & DNA/Polinton \\
\hline
\end{tabular}

CgiSat01a and CgiSat01b are variants (sub-families) of the Cg170/HindIII repeat family described by Clabby et al. [36] and López-Flores et al. [40] and confirmed as the most abundant tandem repeat of this species [35]. A few satDNAs correspond to several 
clusters of Helitron-incorporated tandem repeats reported by Vojvoda Zeljko et al. [37]; in particular, CgiSat01 corresponds to CL1, 2, 10 and 13, CgiSat08 and CgiSat37 to CL 3 and CL 7, respectively, and CgiSat09 to sequences from CL10 and 13. CgiSat17, CgiSat28 and CgiSat46 hold similarity to three clusters of sequences ( $\mathrm{Cl} 112,460,150$, respectively) enriched in the sample of the immunoprecipitated, CenH3-associated DNA sequences of the Pacific oyster [35].

The 52 satDNAs build $6.33 \%$ of the $C$. gigas genome. Consensus sequences of the satDNA monomers were used for screening of Repbase [41], a database holding different types of repetitive sequences and mobile elements. The search revealed that the satDNA sequences constituting $91.76 \%$ of the satellitome show similarity to sequences annotated as different mobile elements. Most of them, $75.61 \%$ of the satellitome, are showing similarity to the central repeats of Helitron mobile elements. For the rest of the mobile elements, the similarity was fragmentary and limited to only a segment of the satDNA monomer sequence.

\subsection{In Silico Chromosomal Localization of C. gigas satDNAs}

The distribution of the 52 satDNAs on the chromosomes of the Pacific oyster was inspected by an in silico analysis. For that purpose we annotated consensus sequences of each satDNA (allowing 70\% similarity to detect different variants of monomer sequence) on the two currently available chromosome assemblies of $C$. gigas. The genome assembly holding acc. number GCA_902806645.1 [39] consists of ten chromosomes (linkage groups LG1-LG10) and 226 unplaced scaffolds, while the assembly under acc. number GCA_011032805.1 reports only ten chromosomes and no additional data. As shown in Figure 2, satDNAs display differences in chromosome placement, dissemination, and monomer grouping. For instance, CgiSat03 is highly interspersed throughout all chromosomes of C. gigas, CgiSat27 is interspersed on 6 chromosomes, while CgiSat22 is limited to a single locus on one chromosome. The in silico chromosomal localization of all satDNAs is presented in Figure S1.

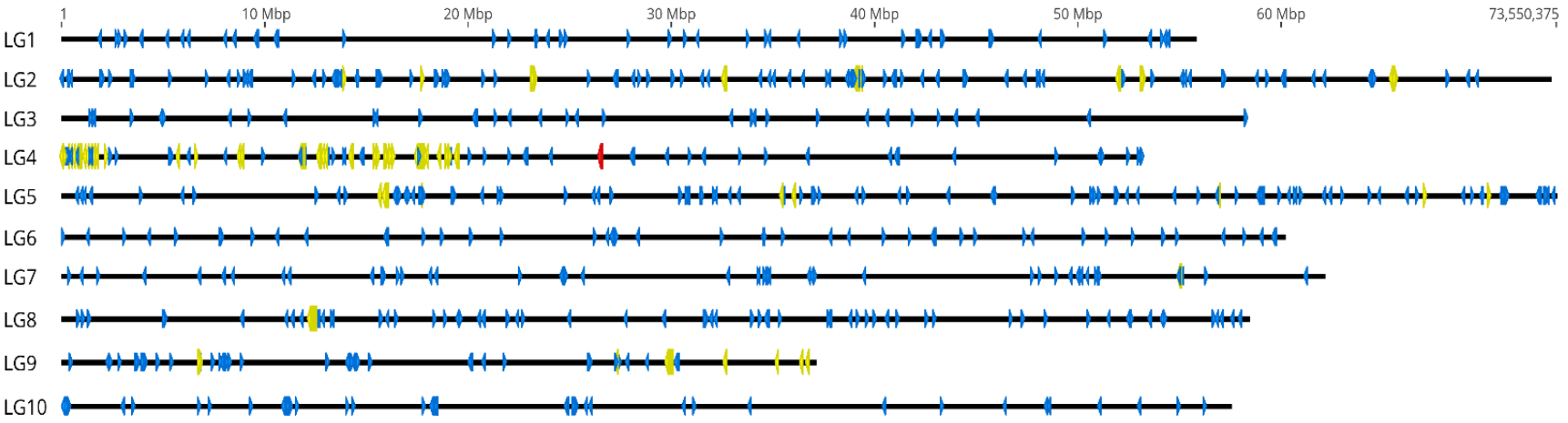

Figure 2. In silico localization exampled by three satDNAs annotated on the chromosomes of the C. gigas currently representative genome assembly GCA_902806645.1. CgiSat03 (blue), CgiSat27 (yellow) and CgiSat22 (red).

Presence or absence of each of the 52 satDNAs on each chromosome were noted for the two genome assembly datasets together with the number of annotated monomers on chromosomes and unplaced scaffolds (Table 2). As CgiSat50 was not detected on any of the chromosomes of this species, an NCBI blast search was performed, revealing that this tandem repeat corresponds to the mitohondrial rDNA sequence. Twenty-five of the 52 satDNAs are widespread and appear on all chromosomes of the Pacific oyster, while the others are restricted to some or even a single chromosome. 
Table 2. Distribution of the 52 satDNAs constituting the satellitome of $C$. gigas on the chromosomes of two genome assembly datasets.

\begin{tabular}{|c|c|c|c|c|c|c|c|c|c|c|c|c|c|c|c|c|c|c|c|c|c|c|c|c|c|}
\hline \multirow{3}{*}{ satDNA } & \multicolumn{13}{|c|}{ Assembly Accession: GCA_902806645.1 } & \multicolumn{12}{|c|}{ Assembly Accession: GCA_011032805.1 } \\
\hline & \multicolumn{9}{|c|}{ LINKAGE GROUP } & \multirow{2}{*}{$\begin{array}{c}\text { No. of } \\
\text { Chromo- } \\
\text { somes } \\
\text { Occupied } \\
\end{array}$} & \multirow{2}{*}{$\begin{array}{c}\text { No. of } \\
\text { Monomers } \\
\text { on Chromo- } \\
\text { somes }\end{array}$} & \multirow{2}{*}{$\begin{array}{c}\text { No. of } \\
\text { Monomers on } \\
\text { Unplaced } \\
\text { Scaffolds } \\
\end{array}$} & \multirow[t]{2}{*}{$\begin{array}{c}\text { Average No. of } \\
\text { Monomers Per } \\
\text { Chromosome }\end{array}$} & \multirow[t]{2}{*}{ satDNA } & \multicolumn{8}{|c|}{ CHROMOSOME } & \multirow{2}{*}{\begin{tabular}{|c|c|c|c|}
$\begin{array}{c}\text { No. of } \\
\text { Chromosomes } \\
\text { Occupied }\end{array}$ \\
10
\end{tabular}} & \multirow{2}{*}{$\begin{array}{c}\text { No. of } \\
\text { Monomers } \\
\text { on Chromo- } \\
\text { somes } \\
\end{array}$} & \multirow[t]{2}{*}{$\begin{array}{l}\text { Average No. of } \\
\text { Monomers Per } \\
\text { Chromosome }\end{array}$} \\
\hline & 1 & 2 & 3 & 4 & 5 & 6 & 8 & 9 & 10 & & & & & & 1 & 2 & 3 & 4 & 56 & 7 & 8 & 10 & & & \\
\hline CgiSat02 & + & + & + & + & + & + & $+\quad+$ & + & + & 10 & 6499 & 1249 & 650 & CgiSat02 & + & + & + & + & + & + & ++ & ++ & 10 & 5563 & 556 \\
\hline CiSat03 & + & + & + & + & + & + & ++ & + & + & 10 & 2502 & 411 & 250 & CgiSat03 & + & + & + & + & $+t$ & ++ & ++ & ++ & 10 & 2116 & 212 \\
\hline CgiSat04 & + & + & + & + & + & + & $+\quad+$ & + & + & 10 & 223 & 32 & 22 & CgiSat04 & + & + & + & + & $+t$ & $+\quad+$ & $+\quad+$ & $+\quad+$ & 10 & 198 & 20 \\
\hline CgiSat05 & + & + & + & + & + & + & $+\quad+$ & + & + & 10 & 1811 & 312 & 181 & CgiSat05 & + & + & + & + & $+t$ & + & $+\quad+$ & $+\quad+$ & 10 & 1553 & 155 \\
\hline CiSat06 & + & + & + & + & + & + & $+\quad+$ & + & + & 10 & 1628 & 202 & 163 & CgiSat06 & + & $\begin{array}{lll}+ & & \end{array}$ & + & + & $+t$ & ++ & ++ & $+\quad+$ & 10 & 1574 & 157 \\
\hline CgiSat07 & + & + & + & - & + & + & $+\quad+$ & + & + & 9 & 55 & 11 & 6 & CgiSat07 & + & + & + & + & $+t$ & $+\quad+$ & $+\quad+$ & $+\quad+$ & 10 & 48 & 5 \\
\hline CgiSat08 & + & + & + & + & + & + & $+\quad+$ & + & + & 10 & 5171 & 1030 & 517 & CgiSat08 & + & + & + & + & $+t$ & + & $+\quad+$ & $+\quad+$ & 10 & 4949 & 495 \\
\hline CgiSat09 & + & + & + & + & + & + & $+\quad+$ & + & + & 10 & 4411 & 406 & 441 & Cgisat09 & + & $\begin{array}{lll}+ & & \end{array}$ & + & + & $+t$ & ++ & ++ & ++ & 10 & 4609 & 461 \\
\hline CgiSat10 & + & + & + & + & + & + & $+\quad+$ & + & + & 10 & 1082 & 328 & 108 & CgiSat10 & + & + & + & + & $+t$ & $+\quad+$ & $+\quad+$ & $+\quad+$ & 10 & 1463 & 146 \\
\hline Cgisat11 & - & + & - & - & + & - & $+\quad-$ & - & - & 3 & 83 & 50 & 28 & Cgisat11 & + & - & + & + & + & -+ & $+\quad-$ & $-\quad-$ & 5 & 150 & 30 \\
\hline CgiSat12 & - & + & + & - & + & + & -+ & + & - & 6 & 244 & 21 & 41 & CgiSat12 & + & $\begin{array}{lll}+ & & \end{array}$ & + & + & + & -+ & ++ & -+ & 8 & 672 & 84 \\
\hline CgiSat13 & - & + & + & + & + & + & $+\quad+$ & + & + & 9 & 39 & 2 & 4 & CgiSat13 & + & + & - & + & + & -+ & $+\quad+$ & $+\quad-$ & 7 & 39 & 6 \\
\hline CgiSat14 & + & + & + & + & + & + & $+\quad+$ & + & + & 10 & 441 & 119 & 44 & CgiSat14 & + & + & + & + & + & $+\quad+$ & $+\quad+$ & $+\quad+$ & 10 & 257 & 26 \\
\hline CgiSat15 & + & + & + & + & + & + & $+\quad+$ & + & + & 10 & 1768 & 163 & 177 & CgiSat15 & + & + & + & + & $+t$ & $+\quad+$ & $+\quad+$ & $+\quad+$ & 10 & 1897 & 190 \\
\hline CiSat16 & - & + & + & + & + & + & $+\quad+$ & + & + & 9 & 66 & 1 & 7 & CgiSat16 & + & $\begin{array}{lll}+ & & \end{array}$ & + & + & -+ & $+\quad-$ & -- & $+\quad-$ & 6 & 34 & 6 \\
\hline CgiSat17 & + & + & + & + & + & + & $+\quad+$ & + & + & 10 & 1619 & 309 & 162 & CgiSat17 & + & + & + & + & + & + & $+\quad+$ & $+\quad+$ & 10 & 1153 & 115 \\
\hline CgiSat18 & + & + & + & + & + & + & $+\quad+$ & + & + & 10 & 363 & 25 & 36 & CgiSat18 & + & + & + & + & $+t$ & $+\quad+$ & $+\quad+$ & $+\quad+$ & 10 & 372 & 37 \\
\hline CgiSat19 & + & + & + & + & + & + & $+\quad+$ & + & + & 10 & 947 & 352 & 95 & CgiSat19 & + & $\begin{array}{lllllll}+ & & l\end{array}$ & + & $\begin{array}{lll}+ & & l\end{array}$ & $+t$ & ++ & ++ & $+\quad+$ & 10 & 1304 & 130 \\
\hline CiSat22 & - & - & - & + & - & - & -- & - & - & 1 & 45 & 0 & 45 & CgiSat22 & - & - & - & - & -- & -- & -+ & -- & 1 & 31 & 31 \\
\hline CgiSat23 & + & + & + & + & + & + & $+\quad+$ & + & - & 9 & 78 & 14 & 9 & CgiSat23 & + & + & + & + & + & $+\quad+$ & + & $+\quad+$ & 10 & 148 & 15 \\
\hline CgiSat24 & + & + & + & + & + & + & ++ & + & + & 10 & 2740 & 128 & 274 & CgiSat24 & + & + & + & + & $+t$ & ++ & + & ++ & 10 & 2829 & 283 \\
\hline CgiSat25 & + & + & + & + & + & + & $+\quad+$ & + & + & 10 & 2468 & 67 & 247 & CgiSat 25 & + & $\begin{array}{lllllll}+ & & l\end{array}$ & + & $\begin{array}{lll}+ & & l\end{array}$ & $+t$ & ++ & + & $+\quad+$ & 10 & 2983 & 298 \\
\hline CgiSat26 & - & - & - & - & - & - & -+ & - & - & 1 & 13 & 0 & 13 & CgiSat26 & - & - & - & + & - & -- & -- & -- & 1 & 15 & 15 \\
\hline CgiSat27 & - & $\begin{array}{llll}+ & & & \end{array}$ & + & + & + & - & $+\quad+$ & + & - & 7 & 2030 & 57 & 290 & Cgisat27 & + & - & + & + & + & -+ & + & -+ & 7 & 1554 & 222 \\
\hline CgiSat28 & + & + & + & + & + & + & $+\quad+$ & + & + & 10 & 487 & 167 & 49 & Cgisat28 & + & $\begin{array}{llll}+ & & & \end{array}$ & + & + & $+\quad+$ & $+\quad+$ & + & $+\quad+$ & 10 & 548 & 55 \\
\hline CgiSat29 & + & + & + & + & + & + & $+\quad+$ & + & + & 10 & 277 & 19 & 28 & CgiSat29 & + & $\begin{array}{lll}+ & & \\
\end{array}$ & + & + & + & $+\quad+$ & + & $+\quad+$ & 10 & 267 & 27 \\
\hline CgiSat30 & - & - & - & + & - & + & -- & - & - & 2 & 2 & 1 & 1 & CgiSat30 & - & - & - & - & + & -- & -+ & -- & 2 & 4 & 2 \\
\hline CgiSat31 & + & - & - & - & - & - & -- & - & - & 1 & 52 & 0 & 52 & CgiSat31 & - & - & - & - & - & -+ & - & -- & 1 & 61 & 61 \\
\hline CgiSat32 & - & + & + & - & + & + & -- & - & - & 4 & 147 & 0 & 37 & CgiSat32 & + & - & - & - & - & -+ & - & -- & 2 & 75 & 38 \\
\hline CgiSat33 & - & + & + & + & + & + & $+\quad+$ & + & + & 9 & 3191 & 254 & 355 & CgiSat33 & - & + & - & + & $+t$ & ++ & + & $+\quad+$ & 8 & 2789 & 349 \\
\hline CgiSat34 & - & + & - & - & - & - & -+ & + & + & 4 & 12 & 2 & 3 & CgiSat34 & + & $\begin{array}{lll}+ & & \\
\end{array}$ & - & + & $+t$ & ++ & + & ++ & 9 & 43 & 5 \\
\hline CgiSat35 & - & + & - & - & + & + & -- & - & + & 4 & 6 & 0 & 2 & CgiSat35 & - & - & + & - & --1 & -+ & - & -- & 2 & 4 & 2 \\
\hline CgiSat36 & + & - & - & - & - & - & -- & - & - & 1 & 2 & 57 & 2 & CgiSat36 & - & - & - & - & + & $+\quad+$ & - & -+ & 4 & 96 & 24 \\
\hline CiSat37 & + & + & + & + & + & + & $+\quad+$ & + & + & 10 & 6367 & 516 & 637 & CgiSat37 & + & $\begin{array}{lll}+ & & \\
\end{array}$ & + & + & + & ++ & + & ++ & 10 & 6242 & 624 \\
\hline CgiSat38 & + & + & - & + & - & + & -+ & - & - & 5 & 6 & 0 & 1 & CgiSat38 & + & - & + & - & -+ & $+\quad-$ & -+ & $+\quad-$ & 5 & 10 & 2 \\
\hline CgiSat39 & + & + & + & + & + & + & $+\quad+$ & + & + & 10 & 1617 & 64 & 162 & CgiSat39 & + & + & + & + & $+t$ & $+\quad+$ & + & $+\quad+$ & 10 & 1235 & 124 \\
\hline CiSat40 & + & + & + & + & + & + & $+\quad+$ & + & + & 10 & 402 & 31 & 40 & CgiSat40 & + & $\begin{array}{lll}+ & & \end{array}$ & + & + & $+t$ & ++ & + & $+\quad+$ & 10 & 402 & 40 \\
\hline CgiSat41 & - & + & - & - & - & - & -- & - & - & 1 & 11 & 0 & 11 & CgiSat41 & + & - & - & - & - & -- & -- & -- & 1 & 7 & 7 \\
\hline CgiSat42 & + & + & + & - & + & + & $+\quad+$ & - & - & 7 & 124 & 31 & 18 & CgiSat42 & + & - & + & + & + & -+ & - & -- & 5 & 104 & 21 \\
\hline CgiSat43 & + & + & + & + & + & + & $+\quad+$ & + & + & 10 & 3145 & 380 & 315 & CgiSat43 & + & $\begin{array}{lll}+ & & \end{array}$ & + & + & + & ++ & + & $+\quad+$ & 10 & 3416 & 342 \\
\hline CgiSat44 & + & + & + & + & + & + & $+\quad+$ & + & + & 10 & 54 & 2 & 5 & CgiSat44 & + & $\begin{array}{lll}+ & & \end{array}$ & + & + & $+t$ & $+\quad+$ & + & $+\quad+$ & 10 & 80 & 8 \\
\hline
\end{tabular}


Table 2. Cont

\begin{tabular}{|c|c|c|c|c|c|c|c|c|c|c|c|c|c|c|c|c|c|c|c|c|c|c|c|c|}
\hline \multicolumn{13}{|c|}{ Assembly Accession: GCA_902806645.1 } & \multicolumn{12}{|c|}{ Assembly Accession: GCA_011032805.1 } \\
\hline \multirow[t]{2}{*}{ satDNA } & \multicolumn{8}{|c|}{ LINKAGE GROUP } & \multirow[t]{2}{*}{$\begin{array}{c}\text { No. of } \\
\text { Chromo- } \\
\text { somes } \\
\text { Occupied } \\
\end{array}$} & \multirow[t]{2}{*}{$\begin{array}{c}\text { No. of } \\
\text { Monomers } \\
\text { on Chromo- } \\
\text { somes }\end{array}$} & \multirow[t]{2}{*}{$\begin{array}{c}\text { No. of } \\
\text { Monomers on } \\
\text { Unplaced } \\
\text { Scaffolds } \\
\end{array}$} & \multirow[t]{2}{*}{$\begin{array}{l}\text { Average No. of } \\
\text { Monomers Per } \\
\text { Chromosome }\end{array}$} & \multirow[t]{2}{*}{ satDNA } & \multicolumn{8}{|c|}{ CHROMOSOME } & \multirow[t]{2}{*}{$\begin{array}{c}\text { No. of } \\
\text { Chromosomes } \\
\text { Occupied }\end{array}$} & \multirow[t]{2}{*}{$\begin{array}{c}\text { No. of } \\
\text { Monomers } \\
\text { on Chromo- } \\
\text { somes }\end{array}$} & \multirow[t]{2}{*}{$\begin{array}{l}\text { Average No. of } \\
\text { Monomers Per } \\
\text { Chromosome }\end{array}$} \\
\hline & 1 & 2 & 3 & 4 & 56 & 67 & $8 s$ & $9 \quad 10$ & & & & & & 1 & 2 & 3 & 45 & 6 & 7 & 8 & 910 & & & \\
\hline CgiSat45 & - & + & - & - & - & -- & - & -- & 1 & 1 & 11 & 1 & CgiSat45 & + & - & - & $-{ }_{-}$ & - & - & - & -- & 1 & 4 & 4 \\
\hline CgiSat46 & + & - & - & - & + & -- & - & -+ & 3 & 25 & 0 & 8 & CgiSat46 & - & + & - & ++ & + & - & + & +- & 6 & 359 & 60 \\
\hline CgiSat47 & + & + & + & - & + & $+\quad-$ & + & +- & 7 & 251 & 79 & 36 & CgiSat47 & $\begin{array}{llll}+ & & & \end{array}$ & - & + & ++ & + & + & + & + & 9 & 340 & 38 \\
\hline CgiSat48 & - & - & + & + & + & ++ & + & -+ & 7 & 20 & 4 & 3 & CgiSat48 & + & + & + & +- & + & - & - & -+ & 6 & 10 & 2 \\
\hline CgiSat49 & + & + & + & + & ++ & $+\quad+$ & + & $+\quad+$ & 10 & 77 & 4 & 8 & CgiSat49 & + & + & + & $+\quad+$ & + & + & + & $+\quad+$ & 10 & 79 & 8 \\
\hline CgiSat50 & - & - & - & - & - & -- & - & -- & 0 & 0 & 0 & 0 & CgiSat50 & - & - & - & $-\quad-$ & - & - & - & -- & 0 & 0 & 0 \\
\hline CgiSat52 & + & - & - & - & -- & -- & - & -- & 1 & 17 & 0 & 17 & CgiSat52 & $\begin{array}{llll}- & & & \end{array}$ & - & - & -- & -- & + & - & -- & 1 & 6 & 6 \\
\hline
\end{tabular}

For each satDNA, presence (+) or absence (-) and total number of monomers present on chromosomes and unplaced scaffolds are indicated. 
The distribution patterns of the satDNAs were also inspected in regard to their connection to the mobile elements reported in Table 1. When the complete pool of mobile element-related satDNAs was checked, they exhibited no advantage in dispersal as the average number of the ocuppied chromosomes was 7.3 for mobile element-related satDNAs, and 7.0 for unrelated ones. However, when only Helitron-related ones were taken into consideration, the average number of ocuppied chromosomes was higher, 9.7 (Figure S2).

Additionally, the satDNAs present on a single chromosome in both assemblies, without hits in the unplaced scaffolds, provided the opportunity to associate some linkage group-based chromosomes of the GCA_902806645.1 dataset to the chromosomes of the GCA_011032805.1 assembly demonstrating that the profiles of CgiSat22, 26, 31, 41 and 52 are shared between LG4 and chr8, LG8 and chr4, LG1 and chr7, and LG2 and chr1 (Table 2).

\subsection{Deciphering the Dominant Organizational Forms of C. gigas Tandem Repeats}

Taking into consideration that a significant part of the C. gigas satellitome shows similarity to Helitron mobile elements (Table 1), we explored what the most common organizational form in which these sequences exist in this genome is: element-associated, standalone or both. Helitron elements usually consist of two well-structured left and right sequence segments (conserved boxes) and of a microsatellite followed by a short array of tandemly repeated satDNA monomers $[17,37,38,42,43]$. Table S2 displays the Helitrons showing similarity to C. gigas satDNAs after Repbase search. Sequence comparisons of those elements revealed high nucleotide sequence similarity in structural segments of some of them, primarily in the regions of the element ends, conserved boxes. Consequently, they can be divided into three groups. Helitron-N2_Cgi and N2C_Cgi share a $44 \mathrm{bp}$ segment at the beginning of the elements (Box 1), a $49 \mathrm{bp}$ segment at their ends (Box 2) and $156 \mathrm{bp}$ between the microsatellite and the central repeats (Box 3). Helitron-N3_Cgi, N4_Cgi, N28_Cgi, N29_Cgi, N31_Cgi, N32_Cgi, N35_Cgi, N40_Cgi share the conserved $53 \mathrm{bp}$ segment at the beginning (Box 4) and $42 \mathrm{bp}$ at the end of the elements (Box 5). Nucleotide sequences of Boxes 1-5 are presented in Table S3. For Helitron-1 DEu, N25_Cgi, N62B_Cgi and N12_Cgi, the conserved boxes could not be determined, as they showed no similarity in terminal sequences among each other nor to the rest of the inspected Helitrons. Satellite DNAs showing similarity to the Helitrons whose conserved segments at the element ends could be determined were used for deciphering their most frequent organizational form. Eleven satDNAs meet this requirement: CgiSat01, 02, 03, 04, 05, 06, $07,09,14,17$ and 48 , together constituting $64.25 \%$ of the satellitome. For that purpose, after the annotation of sequences of these satDNAs on chromosomes and scaffolds, the surrounding of each satDNA sequence (of at least one complete monomer), was inspected for the presence of conserved segments belonging to Helitron mobile elements. SatDNA sequences together with $2000 \mathrm{bp}$ of left and right flanking regions were extracted from the currently representative chromosome assembly GCA_902806645.1 and searched for the presence of Boxes $1 \& 2$ or Boxes $4 \& 5$ that designate the element ends. The results are presented in Table S4. If the corresponding boxes were detected at each side of the repeat within the extraction, they were classified as element-associated. Structures having a box only on one side of the repeats were also included in this category, as truncation on one side of these elements is a rather frequent event [44]. If conserved boxes were not detected in the surrounding segments, repeats were classified as standalone.

The main organizational forms of 11 satDNAs are depicted in Figure 3. Nine of them present mobile element association as dominant occurrence form, ranging from 69.9 (CgiSat14) to $100 \%$ (CgiSat48) of the extractions. Only CgiSat48 is exclusively mobile element-associated. Two satDNAs were dominantly found in standalone forms, CgiSat04 (78.77\% of the extractions) and CgiSat07 (96.00\%). 

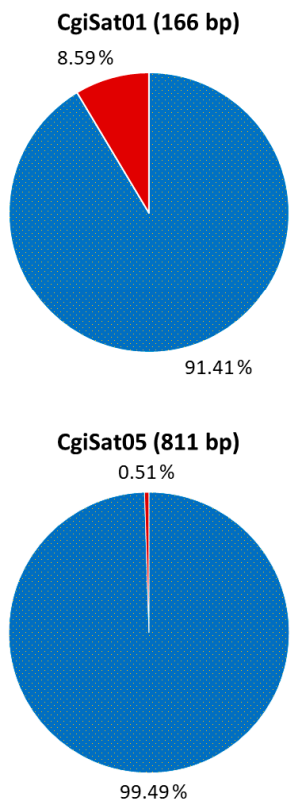

CgiSat14 (1144 bp)

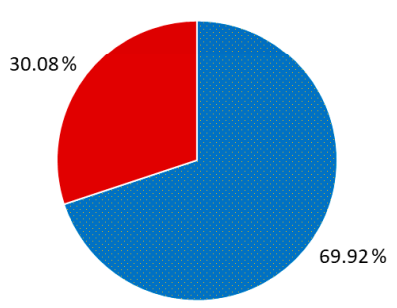

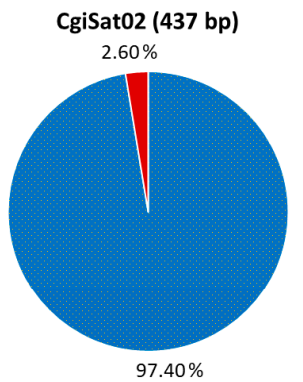

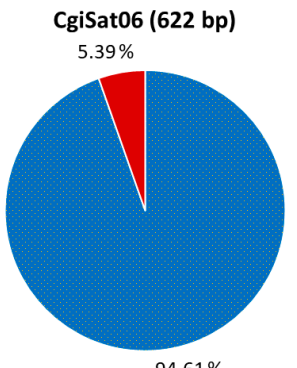

CgiSat17 (134 bp)

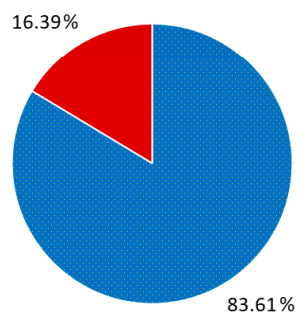

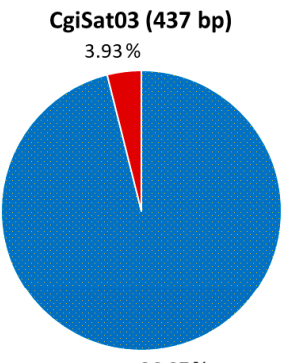

$96.07 \%$

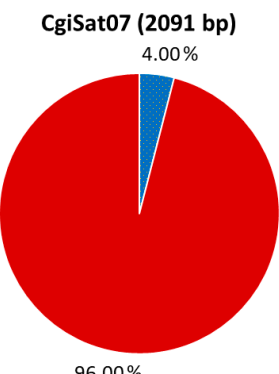

CgiSat48 (2056 bp)

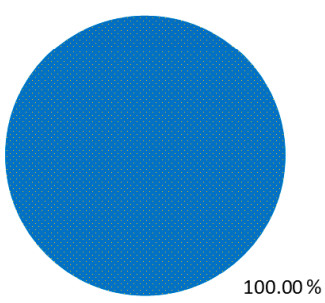

CgiSat04 (2077 bp)
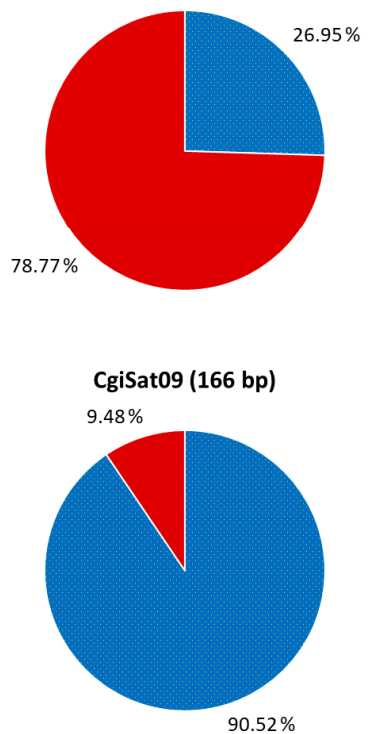

- element-associated - standalone

Figure 3. Proportions (\%) of mobile element-associated (blue) and standalone (red) organizational forms in extractions corresponding to the satDNAs of C. gigas. Monomer sizes are provided in parentheses.

The number of monomers and the presence of the boxes for each satDNA were also analyzed for every extraction. Interestingly, single monomers, regardless of whether they are element-associated or standalone, were the most abundant category of extractions for the majority of the satDNA sequences, with the exception of CgiSat02 and CgiSat17 (Figure 4).

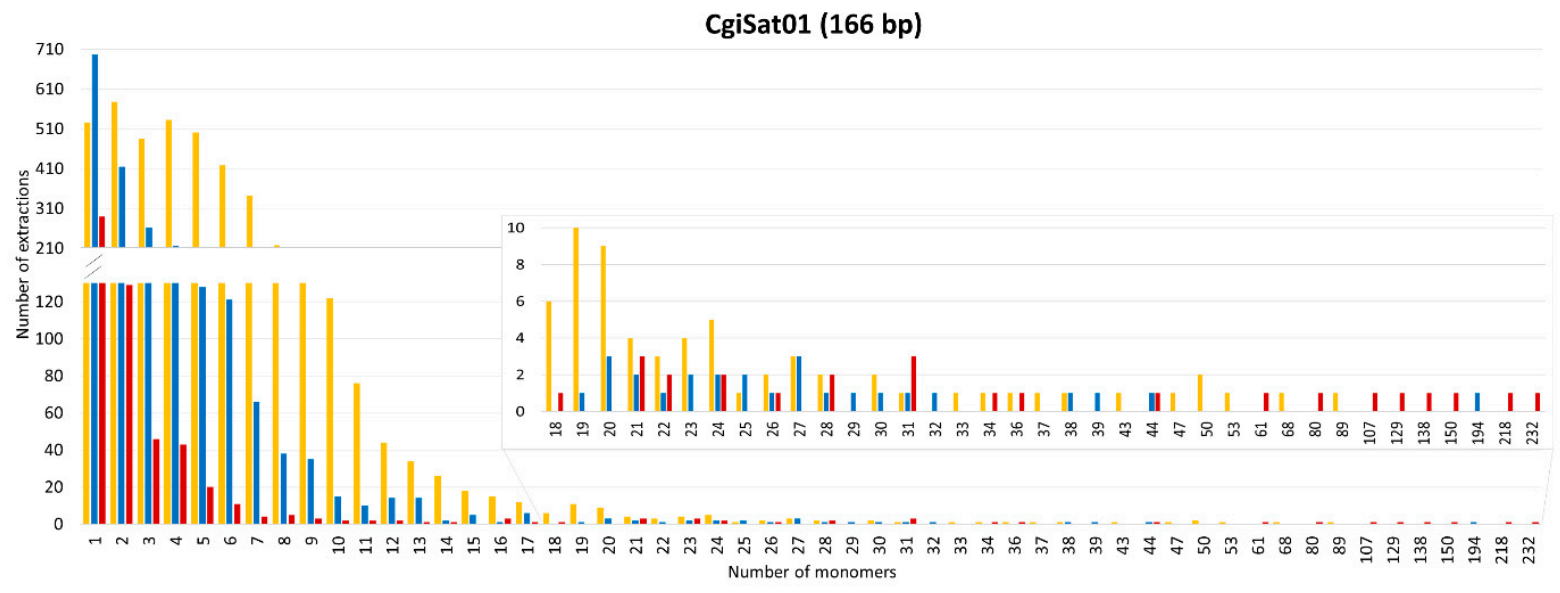

Figure 4. Cont. 

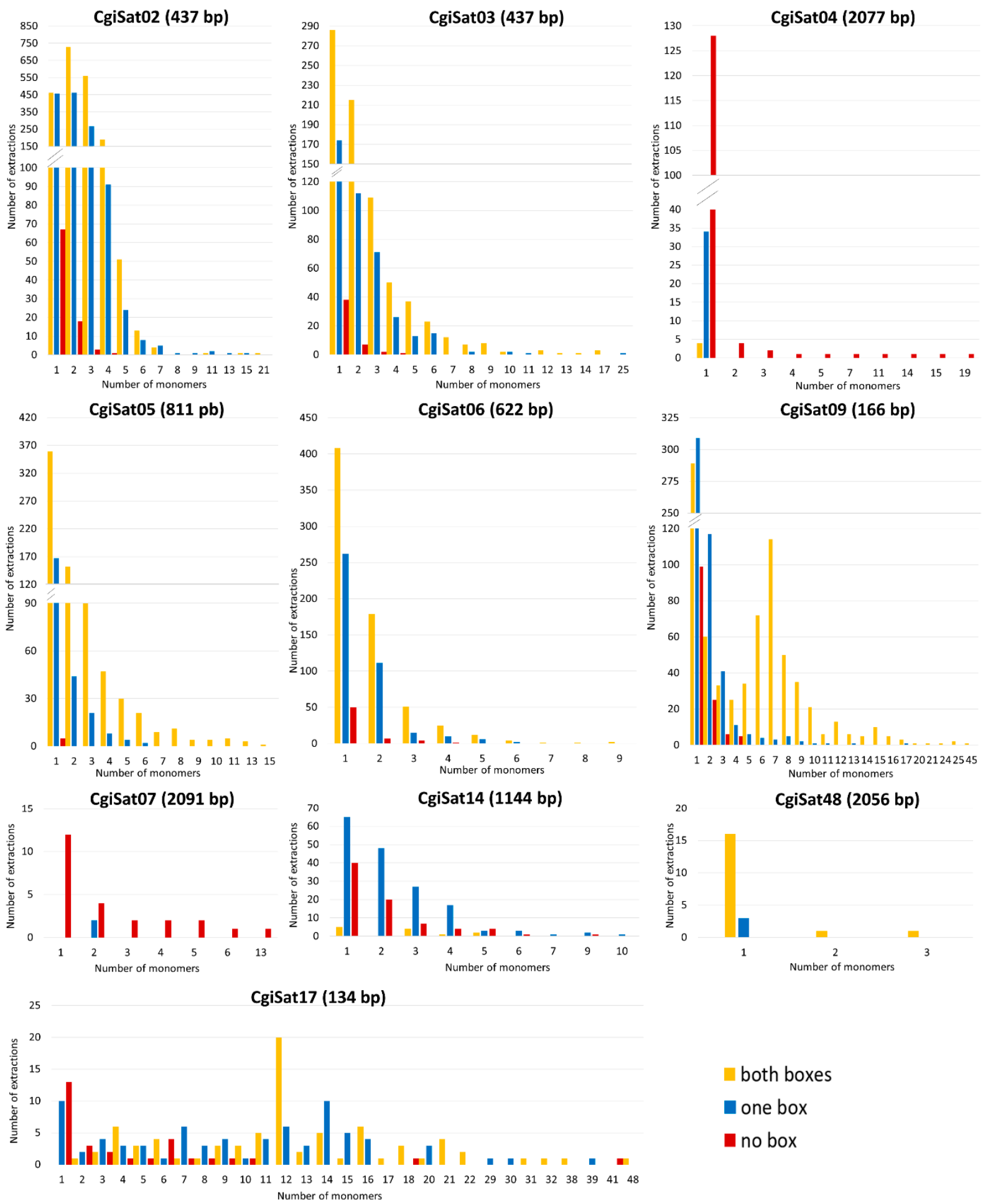

Figure 4. Number of satDNA monomers per extraction and number of extractions for the eleven closely inspected satDNAs of $C$. gigas. Presence or absence of Helitron boxes flanking satDNAs are presented by yellow (Helitron boxes at both sides), blue (Helitron boxes at one side) and red (no Helitron boxes) bars.

The most abundant satDNA of $C$. gigas, CgiSat01, presented the largest number of extractions and longest arrays of tandem repeats. For this satDNA the longest mobile element-associated array has 89 monomers while the longest standalone one is built of 232 monomers (about $40 \mathrm{~kb}$ ). The latter array was found to hold an assembly gap, thus potentially forming an even longer stretch in the genome on LG3. Although characterized 
as a satDNA in the RepeatExplorer2 TAREAN analysis, arrays with more than three tandemly arranged repeats were not detected for CgiSat48. Its dominant form are single monomers with Helitron boxes present at both ends. It should be noted that CgiSat 48 monomer repeats are unusually long, about $2056 \mathrm{bp}$, and composed of the unique sequence that could not be resolved into potential subunits. For the rest of the inspected satDNAs, the maximum number of monomers in an array ranged from nine (CgiSat06) to 48 (CgiSat17). Maximum array length and mobile element-association do not seem to be interdependent. Namely, for all but CgiSat01 inspected satDNAs the longest array belongs to the dominant organizational form of the corresponding satDNA, regardless if it is mobile elementassociated or standalone (Figure 4). It was also observed that the satDNA monomer size and the maximum number of monomers that can be found in an array are not interdependent (Figure S3).

\subsection{Fluorescence In Situ Hybridization}

The chromosomal distribution of the most abundant satDNA, CgiSat01, was already described by Wang et al. [45] and Tunjić Cvitanić et al. [35]. It displayed strong, discrete FISH signals in the centromeric regions of several chromosomes of $C$. gigas, together with highly interspersed signals on chromosome arms. Here, we performed fluorescence in situ hybridization on metaphase chromosomes for several of the most prominent satDNAs of the Pacific oyster (Figure 5a-i).

CgiSat02 and CgiSat09 present a substantial number of signals along chromosome arms. CgiSat03, 04, 05, 17 and 37 exhibit similar interspersed pattern but with reduced number of signals, which is even more limited for CgiSat28 and CgiSat46. To confirm the specificity of such signal distribution, a few satDNA probes were hybridized together with $5 \mathrm{~S}$ rDNA probes as a control (Figure $5 \mathrm{~b}-\mathrm{e}, \mathrm{h}$ ). $5 \mathrm{~S}$ rDNA is known to be present on chromosomes 4 and 5, exhibiting strong subtelomeric signals on one chromosome pair and weak ones on another [46], and the same distribution pattern is also noticeable in Figure 5j. 
a

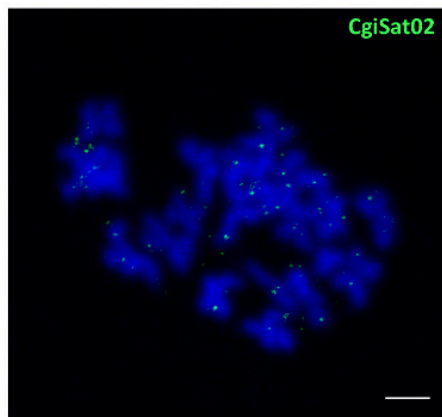

c

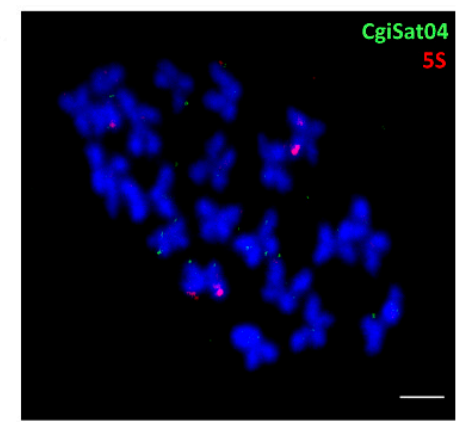

e

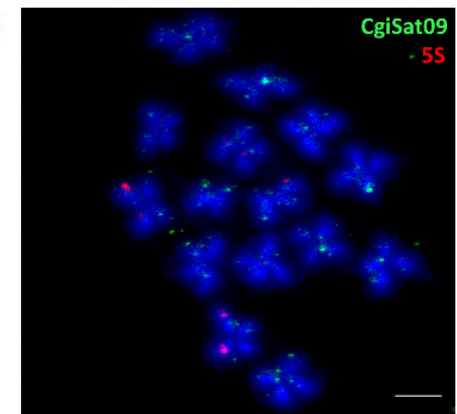

g

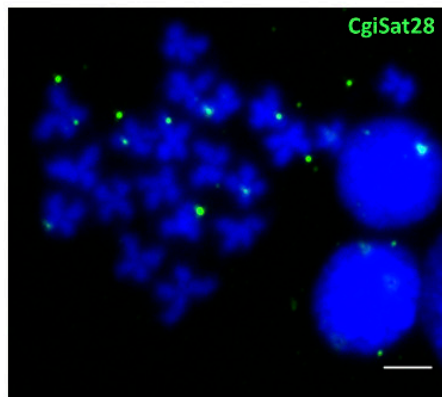

i

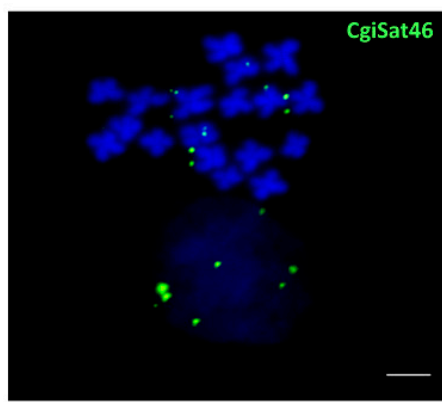

b

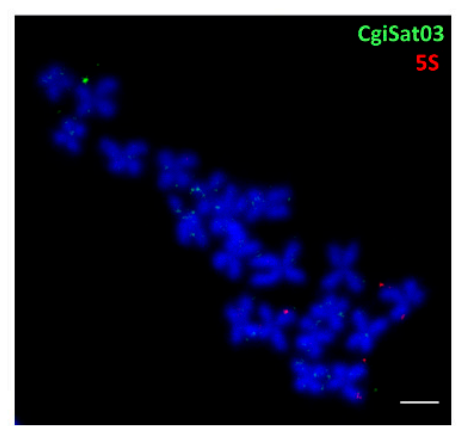

d
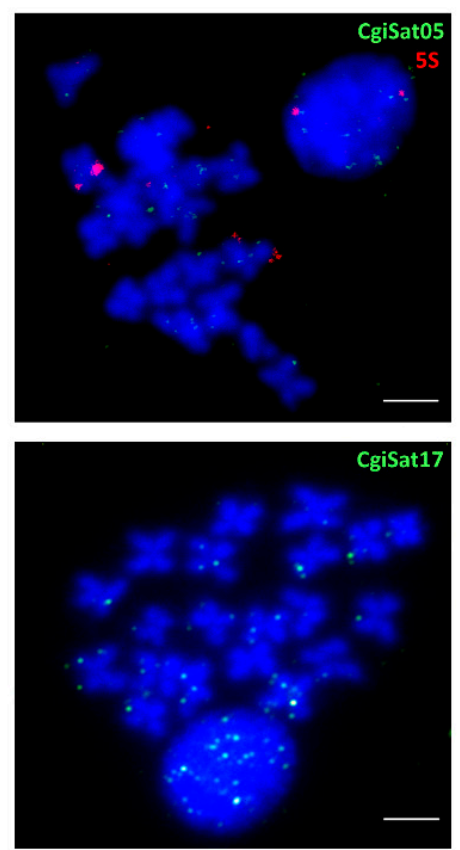

h

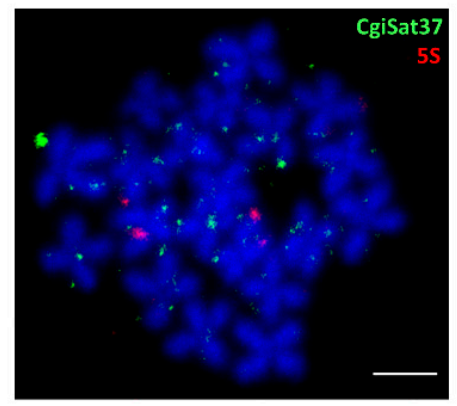

j

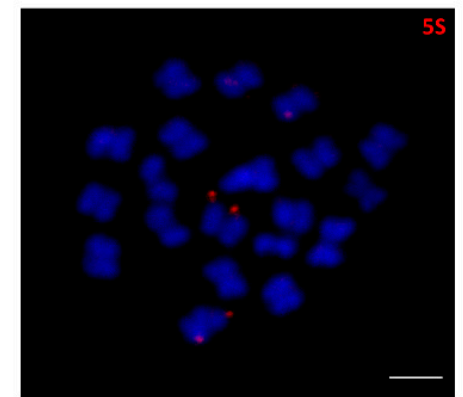

Figure 5. FISH localization of satDNA repeats belonging to (a) CgiSat02 (b) CgiSat03, (c) CgiSat04, (d) CgiSat05, (e) CgiSat09, (f) CgiSat17, (g) CgiSat28, (h) CgiSat37 (i) CgiSat46. To confirm the specificity of the interspersed signal $5 \mathrm{~S}$ rDNA (j) was used as a positive control and co-hybridized with several satDNAs (b-e,h). Scale bars represent $3 \mu \mathrm{m}$. 


\section{Discussion}

In this work we present the satellitome of the Pacific oyster C. gigas, analyse the links between satDNAs and mobile elements, and define some general organizational features of the most prominent repetitive DNAs in this genome. Comprehensive satellitome studies performed recently on diverse species by using advanced methodological approaches highlighted not only the extraordinary diversity in composition and content of satDNAs within and among species but also indicated the sharp contrasts in their genomic arrangements $[4,47]$.

Several characteristics qualify $C$. gigas as a valuable non-standard model species in exploring satellitome and repeatome organizational patterns: the low abundance of satDNAs vs. mobile elements [31,39], the low amount of heterochromatin, limited to the centromeric region of one chromosome pair and the telomeric region of another [35,48], the incorporation of short satDNA arrays into mobile elements of the Helitron/Helentron family [22,37], and the remarkably high number of predicted Helitron-related sequences [39]. C. gigas is also the first bivalve species in which the DNA composition of the centromeric regions and the heterochromatin was explored by using chromatin immunoprecipitation, revealing a predominance of DNA transposons and the lack of centromere-specific repetitive sequences [35].

The introduction of third generation sequencing methods, supplemented with novel mapping and bioinformatic tools, enabled reading long segments composed of satDNAs and filling the unassembled gaps left in earlier genome outputs, populated mostly by sequences repeated in tandem $[49,50]$. The sequencing and assembly of $C$. gigas genome is particularly demanding because of the high level of heterozygosity and the abundance of repetitive sequences. In the first release, those hindrances were solved by a combination of NGS, fosmid pooling, and hierarchical assembly [31,51]. More recently, the de novo sequencing and assembly of the $C$. gigas genome, employing a combination of high coverage long and short read data and linkage maps, resulted in a less fragmented genome and gained two assemblies at the chromosome level, GCA_902806645.1 [39] currently being the representative one.

In this work, the repetitive DNA content of $C$. gigas was accessed by low-coverage NGS followed by RepeatExplorer2 clustering. The detection of 52 satDNAs constituting the satellitome of the Pacific oyster (Table 1) is a giant leap in the knowledge about the satDNA content of this species, up until now limited to only three satDNAs (reviewed in [34]). The employment of NGS methods has immensely improved satDNA detection substantially increasing the number of satDNAs detected in the genomes of many species (e.g., $[9-11,52,53])$. Although satDNA monomer lengths vary significantly in the satellitome of the Pacific oyster, 160-180 bp-long monomers predominate. This monomer size, reflecting nucleosomal periodicity, is generally considered to be evolutionarily favored [54]. The same range of monomer sizes was also observed for a group of short satDNA arrays of C. gigas tandem repeats incorporated in Helitron/Helentron mobile elements [37]. Close connections between satDNAs and mobile elements have been observed in many forms and in many organisms (reviewed in [55]), including bivalves (reviewed in [34]). However, our work evidences an additional level of how vast and intimate the relation between tandem and interspersed repeats can be, as a large part of the C. gigas satellitome exhibits similarity to different mobile elements, especially to those of the Helitron type (Table 1).

As mobile elements were proposed to generate complex rearrangements and even facilitate genomic dispersal of satellite repeats $[17,19,43,56-58]$, it could reasonably be expected that satDNAs connected to mobile elements would have some propagation and dissemination advantages. In C. gigas, only Helitron mobile element-related satDNAs populated higher number of chromosomes when compared to other satDNAs (Figure S2). The rest of the mobile element-related satDNAs present similarity to mobile elements only in parts of their monomer sequence, not being their constitutive part, thus making active propagation of these satDNAs via mobile elements unlikely. Monomers with such fragmentary similarities could be the result of the tandemization of a segment of the mobile 
element and a nearby sequence, the imprecise excision of mobile elements leaving behind some sequence segments, or some other sequence rearrangements. On the other side, the substantial contribution of the Helitron-related satDNAs to the satellitome of the Pacific oyster $(75.61 \%)$ speaks in favor of these mobile elements being the main players in satDNA propagation in this organism.

However, the final number of chromosomes occupied by the mobile element-related and unrelated satDNAs has to be taken with reservations, as unplaced scaffolds still exist for the currently representative genome assembly, potentially broadening the span of chromosomes occupied by one or both groups of satDNAs.

After determination of the conserved boxes located at the ends of several Helitron elements related to 11 satDNAs, we were able to inspect what the dominant organizational form of those satDNA sequences are: element-associated, standalone, or present in both organizational forms. Interestingly, only CgiSat48 satDNA shows exclusively one organizational form, always being associated with a Helitron (Figure 3). One organizational form prevails in the remaining satDNAs, mobile element-association in eight out of eleven and standalone forms in two. The same sequence can obviously (co)exist in different organizational forms throughout the genome. Such lack of uniformity, and the parallel existence of several different organizational patterns presented by the 11 satDNAs, would suggest that the present complex organization of $C$. gigas tandem repeats is not a result of a single mechanism.

Such unusual organization of satDNAs, largely presenting tandem repeats within mobile elements and only a fraction existing as standalone arrays (Figure 3), significantly differ from the "classical" satDNA organization in long arrays, reported within a wide spectrum of organisms throughout the animal and plant kingdom (humans, insects, and plants; reviewed in [4]). In the special organizational form described in C. gigas only indications of classical satDNAs exist, while most of the tandem repeats are scattered throughout the genome (Figure S1 and Figure 5) without any significant grouping that would clearly distinguish heterochromatic and euchromatic genome compartments. This fact is complementary to the scarceness of heterochromatin in the Pacific oyster, limited to two small, (peri)centromeric and telomeric, segments on two chromosome pairs [35,48].

In regard to the mechanisms leading to such a distribution, several models for forming satDNA arrays from repeats present within mobile elements have been proposed. According to the model proposed by Hikosaka and Kawahara [59] for the satDNA formation from a Miniature Inverted-repeat Transposable Element (MITE), the occurrence of tandem repeats within the MITE element involves the formation of a stem-loop structure between two adjacent MITE elements on the single-stranded DNA, due to the delay of the DNA replication on one strand. This loop is cut out by a nuclease and the remaining strands are rejoined. As a consequence, the new MITE contains repeats from both previous elements. Further extension of the sequence could be accomplished by the same process. Although such sequences are still interspersed repeats, recombination processes could subsequently happen, and the sequences develop into longer arrays of tandem repeats and ultimately into satDNAs. Izsvak et al. [60] also proposed a mechanism based on a stem-loop structure to explain the formation of tandem repeats from a mobile element. During the replication of the MITE element, inverted repeats or palindromic sequences allow forming a stem-loop in the newly synthetized strand while still in the process of synthesis. Then, the whole structure is twisted back, and DNA synthesis continues at the $3^{\prime}$ end of the stem-loop, using the nascent strand as a new template. The duplicated segment is released in the form of an extrachromosomal stem-loop that is incorporated into a new site in the genome, facilitated by the local homology between the motifs in the target sequence and in the amplified extrachromosomal sequence. Structures in MITEs that enable formation of such stem-loop structures, like terminal inverted repeats, are also found at the end of Helitron elements and their structural variants, Helentrons [38].

Furthermore, Helitron/Helentron elements show additional mechanisms involved in their propagation and the amplification of the sequences within. They are known to 
capture segments of the host genome [38], frequently tandem repeats ([22,42,43,61-63], etc.). Helitrons transpose using a rolling circle replication (RCR) mechanism that initiates at the $5^{\prime}$-end and progresses towards the $3^{\prime}$-end, where the $3^{\prime}$ terminal hairpin structure serves as a recognition site for termination and cleavage [44]. Alternatively, during the replication, the original $3^{\prime}$ terminator can be deleted from the circular DNA template by an intramolecular recombination event between internally repeated $5^{\prime}$-ends. The next round of replication generates a tandem array of truncated Helitrons lacking $3^{\prime}$-ends. In the last step, the amplified single complete Helitron copy or multiple truncated Helitron copies are integrated into new genomic locations [44]. Such mechanism could also explain a large number of extractions found in our study, where satDNA sequences were found to be associated with Helitron box only on one side of an array/monomer (Figure 4). Alternatively, such arrays associated to only one box could also result from recombination events between element-associated and standalone arrays, generating hybrid structures. Further prolongation or shortening of the arrays, both element-associated and standalone, could happen via the usual mechanisms of unequal crossing-over exchanges, known to govern satDNA evolution, including repeated rounds of rolling circle replication and reinsertion (reviewed in [3]).

It is also possible that mobile element-associated and standalone forms of satDNA sequences of the Pacific oyster are interchangeable through the interplay of all previously mentioned mechanisms. Related to this, Scalvenzi and Pollet [57] explained two possible directions in the life of the tandem repeats. They propose that precursor satDNA sequences can be captured by a mobile element, followed by the amplification of tandem repeats within. Transposition of elements containing tandem repeats continues but, as the number of repeats within the element increases, the transposition rate of the element decreases. At the same time, recombination rates start to increase with the growing number of monomers, thus causing further expansions of the tandem repeats. Finally, mobile element-associated tandem repeats can give rise to the classical satellite DNA arrays, devoid of surrounding sequences by accumulating mutations over time.

CgiSat01 (corresponding to HindIII/Cg170 satDNA) could be an example for such a scenario. Mobile elements with one to ten internal repeats of CgiSat01 satDNA are present in a large number of copies in the genome due to their continuous transposition, which seems to decay as the number of internal repeats increases. Finally, arrays with more than 100 monomers are dominantly present in a standalone form (Figure 4).

Interestingly, single monomers were the most common extraction for 11 satDNAs inspected, with each satDNA having an individual ratio among extractions surrounded with 2, 1 or 0 mobile element-derived boxes (Figure 4). Such sequences represent the starting- and the end-point of the Scalvenzi and Pollet perpetual model [57], which could potentially be the reason for the large number of extractions holding monomers. Observed structures could have several potential origins. Monomers surrounded with 2 boxes could be a starting structure with possibility of array expansion, or generated after array reductions, while monomers surrounded by only one box or by no boxes could be a result of recombination events, excision events, array reductions, or box deterioration.

Recent studies of satDNA array organization from long-read sequencing data also presented different organization patterns in other organisms. In the plant Lathyrus sativus, 11 major satDNAs showed interesting differences between the analyzed repeats [24]. There, only two satDNAs were predominantly organized in long arrays typical for satDNA, while the remaining nine satDNAs were found to be derived from short tandem arrays located within LTR-retrotransposons, occasionally expanding in length. Likewise, in the C. gigas satellitome, if array length is taken into consideration, only one satDNA (CgiSat01) would be a candidate for a classical satDNA. However, $91.4 \%$ of the extractions holding this sequence were found to be mobile element-associated (Figure 3). On the other hand, two other satDNAs, CgiSat04 and CgiSat07, are dominantly in standalone form, yet their array lengths do not exceed 19 and 13 monomers, respectively, and their monomer sizes exceed $2000 \mathrm{bp}$. Although the most abundant satDNAs were studied in our work, the 
presence of classical satDNAs in the unstudied parts of the satellitome is still possible. However, in that case, the contribution of such sequences to the genome would be very limited, as the abundance of those satDNAs is very low. On the other hand, the detailed examination of the sequences constituting the centromeric and the heterochromatic genome components performed after chromatin immunoprecipitation with anti-CenH3 and antiH3K9me3 antibodies in C. gigas is in line with the above discussed. Sequences building the centromeres were found to be quite heterogeneous and presented high dispersal throughout the genome, while the heterochromatin exhibited general paucity and was predominantly constituted of DNA transposons [35].

\section{Materials and Methods}

\subsection{Sequencing and Read Clustering}

Genomic DNA was extracted from adductor muscle tissue using the DNeasy Blood and Tissue Kit (Qiagen, Hilden, Germany) according to the protocol provided by the manufacturer. As oysters display high levels of phenotypic plasticity, DNA barcoding was performed for molecular identification and species confirmation. For that purpose, primers for the mitochondrial cytochrome c oxidase subunit 1 (COI) gene were used, LCO-1490 5'-GGT CAA CAA ATC ATA AAG ATA TTG G-3' and HCO-2198 5'-TAA ACT TCA GGG TGA CCA AAA AAT CA-3'. PCR amplification was performed with an initial denaturation at $94{ }^{\circ} \mathrm{C}$ for $5 \mathrm{~min}, 35$ cycles of $94{ }^{\circ} \mathrm{C}$ for $30 \mathrm{~s}, 52{ }^{\circ} \mathrm{C}$ for $30 \mathrm{~s}, 72{ }^{\circ} \mathrm{C}$ for $30 \mathrm{~s}$, with a final extension at $72{ }^{\circ} \mathrm{C}$ for $10 \mathrm{~min}$. PCR products were sequenced and compared with publicly available COI sequences of $C$. gigas from NCBI GenBank. Library preparation and Nextgeneration Illumina sequencing of $\mathrm{C}$. gigas genomic DNA was performed on a HiSeqX platform by Admera Health facility (South Pleinfield, NJ, USA). Low-coverage sequencing was implemented, as significantly reduced genome coverage has been recommended for repetitive DNA analysis, due to their enrichment in respect to single-copy ones [64]. C. gigas genome was sequenced to about $1.5 \times$ coverage, generating $2 \times 2,768,912$ paired-end reads, $151 \mathrm{bp}$ in length. Raw sequence reads can be found in NCBI under the BioSample accession number: SAMN15184427, BioProject: PRJNA638244.

Genomic repeat identification was performed using the RepeatExplorer2 pipeline [65] on the Galaxy server (https://repeatexplorer-elixir.cerit-sc.cz/galaxy/, accessed on 1 September 2020). For that purpose, genomic reads were quality-filtered, trimmed, interlaced and paired-end reads with no overlap were further processed. Similarity-based read clustering was performed under the default parameters, using several randomly subsampled sets: two of one million reads, one of two million reads and one set of 1,779,522 reads, corresponding to genome coverages of $0.2 \times, 0.4 \times$ and $0.35 \times$, respectively.

\subsection{Satellite DNA Analysis}

TAREAN [64] incorporated into RepeatExplorer2 pipeline provided the consensus sequences of satDNA monomers. Consensus sequences of satDNAs obtained by four rounds of read clustering were compared to each other using discontinuous megablast with the default parameters in Geneious prime v. 2019.0.4 (Biomatters Ltd., Auckland, New Zealand) in order to detect clusters belonging to the same satDNA in different analyses. The same program was used for all subsequent sequence analysis and editing. Consensus sequences of the 52 satDNAs constituting the satellitome of $C$. gigas are available as Data S1. For the annotation of the 52 satDNAs, two publicly available chromosome-level assemblies of the Pacific oyster genome were downloaded from NCBI, GenBank assembly accession: GCA_902806645.1 [39] and GCA_011032805.1. Consensuses of monomer sequences were used for annotation of each satDNA on chromosomes and scaffolds, allowing 70\% divergence to the consensus in order to encompass different sequence variants.

\subsection{Analysis of the Flanking Regions of the satDNA Arrays}

For the analysis of the flanking regions of the satDNA arrays and single monomers, the currently representative genome assembly GCA_902806645.1 [39] was used. It also 
contains the unplaced scaffolds, genome segments that are usually left unassembled, as they are particularly enriched in sequences repeated in tandem. The .csv file holding a list of exact positions of each annotated monomer for all identified satDNAs was exported from Geneious program. Left and right flanking regions, $2000 \mathrm{bp}$ in length each, were excised along with the surrounded satellite DNA array or single monomer. For excision, we used a custom-made Python script (file parser.py). Input files for Python script were a .csv file containing the chromosome sequence and the file exported from Geneious, with the exact position of the monomers on each chromosome. The Python script output file was a fasta file of the extractions and a .csv file with the information regarding the position of a satellite sequence and the position of the $2000 \mathrm{bp}$ of its left and right flanking regions. The excision holding flanking regions and satDNA array/single monomer was then used for annotation of boxes of interest using Geneious Prime software.

To generate a summary list of the boxes present in the flanking regions of each satDNA array/single monomer, another custom-made Python script was made (file boxer.py). The input file for this Python script was again a Geneious-generated .csv file, holding the information regarding the position of the annotated boxes in the flanking regions. In the Python script output table, the presence of a certain box within the flanking region of the satDNA array/single monomer was marked with 1, and the absence of a box was marked with 0 . The same principle was applied for the excisions of satellite arrays and belonging flanking regions localized in scaffolds and for the detection of the boxes in the scaffold extractions (file parser_scaffolds.py, and file boxer_scaffolds.py). The extractions and annotations were additionally checked by eye. All scripts used are available at: https://bitbucket.org/MonikaTC/tunjic-cvitanic-et-al.-2021/src/master/ (uploaded 1 May 2021).

\subsection{Mitotic Chromosomes Preparations}

Juvenile specimens of the Pacific oyster were collected in Ria de Aveiro, Portugal. Laboratory tanks at $18 \pm 1{ }^{\circ} \mathrm{C}$ with aerated and filtered seawater were used to feed the oysters with microalgae for seven days, in order to promote their growth and maturation. The mitotic chromosome preparations were obtained according to the protocol described in Martinez-Exposito et al. [66], with few modifications. Gills were excised, after a $12 \mathrm{~h}$ treatment of the specimens in a $0.005 \%$ colchicine solution. Hypotonic shock in 50\% and $25 \%$ seawater ( $25 \mathrm{~min}$ each) was performed on gill tissue, followed by fixation in ethanol:acetic acid (3:1) for $1 \mathrm{~h}$. Cell suspensions, obtained by exposing dissected gills to $60 \%$ acetic acid, were dropped onto slides preheated to $56^{\circ} \mathrm{C}$.

\subsection{Probe Labelling}

Probes for fluorescence in situ hybridization corresponding to CgiSat02, 03, 04, 05, $09,17,28,37,46$ and $5 S$ rDNA were labeled by PCR. The reactions contained $50 \mathrm{ng}$ of DNA, $100 \mu \mathrm{M}$ dATP, dGTP and dCTP, $65 \mu \mathrm{M}$ dTTP, $2.5 \mathrm{mM} \mathrm{MgCl2}$, 2.5 U GoTaq G2 Flexi Taq DNA polymerase, $1 \times$ GoTaq Flexi Reaction Buffer (all Promega, Madison, WI, USA), primers ( $1 \mu \mathrm{M}$ each) and $35 \mu \mathrm{M}$ biotin-16-dUTP (Jena Bioscience, Jena, Germany) for satDNAs or $35 \mu \mathrm{M}$ digoxigenin-16-dUTP (Roche, Basel, Switzerland) for $5 \mathrm{~S}$ rDNA, in $50 \mu \mathrm{L}$ volumes. Nucleotide sequences of each primer pair used and PCR amplification conditions employed are presented in Table S5. Probe purification was performed using the QIAquick PCR Purification Kit (Qiagen, Hilden, Germany), following the protocol within. Probes were checked on $1 \%$ agarose gel and the concentration of the purified probes was measured using a Qubit Fluorometer. $30 \mathrm{ng}$ of probe was used per FISH experiment.

\subsection{Fluorescence In Situ Hybridization}

Experiments were performed according to the protocol described in Pérez-García et al. [67], with the alteration in pepsin digestion $\left(5 \mathrm{~min}\right.$ at $\left.37^{\circ} \mathrm{C}\right)$. Prior to usage, DNA probes were denatured at $80^{\circ} \mathrm{C}$ for $8 \mathrm{~min}$ and placed on ice for $2 \mathrm{~min}$. Fluorescein-labelled avidin D and biotinylated anti-avidin D (both Vector Laboratories, Burlingame, CA, USA) 
were used in the signal detection process for biotin-labelled probes and anti-digoxigeninrhodamine Fab fragments (Roche, Basel, Switzerland) for the digoxigenin-labelled probe. Counterstaining of chromosomes was performed using $100 \mathrm{ng} / \mathrm{mL} \mathrm{4',} \mathrm{6-diamidino-2-}$ phenylindole (DAPI) (Sigma-Aldrich, St. Louis, MO, USA), and slides were subsequently mounted in Mowiol 4-88 antifade mounting medium (Sigma-Aldrich, St. Louis, MO, USA). For slide visualization and image capturing Nikon Eclipse-800 fluorescence microscope and a Leica TCS SP8 X laser-scanning microscope were employed.

\section{Conclusions}

The many peculiarities in genome organization already known for C. gigas were furthered in our satellitome analysis. We combined RepeatExplorer2 analysis with the assignment of the obtained sequences on two recent chromosome-level assemblies of C. gigas, followed by FISH localization of the most prominent satDNAs. The satellitome of $C$. gigas is composed of 52 sequences repeated in tandem that altogether build about $6.33 \%$ of the genomic DNA. SatDNAs are distributed along whole chromosomes presenting unusual interspersed patterns, with density and chromosomal distribution specific for each satDNA. In contrast with the established concept of satDNA genomic organization, no significant accumulation of satDNAs was observed in any preferred chromosomal position. Most arrays are relatively short and can be found either as standalone arrays or associated with conserved boxes characteristic for Helitron mobile elements that flank the arrays from one or both sides. Most of the inspected satDNAs are dominantly found in mobile elementassociated form, but two of them mostly appear in a standalone form. Only one of the inspected repeats is present exclusively as element-associated. An advantage in the number of chromosomes occupied was observed for Helitron element-related satDNAs, speaking in favor of satDNAs in C. gigas being propagated with the aid of this family of mobile elements. No evident link between monomer length and the maximum number of monomers that can be found in an array was observed, and the longest array usually belongs to the dominant organizational form of that satDNA, regardless if it is element-associated or standalone. The lack of classical satDNAs in the pool of inspected satDNAs, the lack of uniformity in the organization, and the parallel existence of different organizational patterns within the satellitome, establishes $C$. gigas as a model organism of interest for further detailed studies of repetitive DNA biology.

Supplementary Materials: The following are available online at https://www.mdpi.com/article/10 .3390/ijms22136798/s1.

Author Contributions: E.Š.-V. and M.P. designed the experiments; M.T.-C. and E.Š.-V. performed bioinformatic analyses; M.T.-C. did the experimental work; M.T.-C., J.J.P., D.G.-S. performed the FISH experiments; T.C. wrote the Phyton scripts; M.T.-C., E.S..-V., and M.P. analyzed the data; M.P. and E. S. $-\mathrm{V}$. supervised the research; E.S..-V. prepared the first draft of the manuscript; all authors reviewed and edited the manuscript. All authors have read and agreed to the published version of the manuscript.

Funding: This work was supported by the following projects: Croatian Science Foundation, grant number IP-2019-045522; Xunta de Galicia (ED431C 2020/05) and the European Union (European Regional Development Fund-ERDF); Xunta de Galicia (Centro singular de investigación de Galicia, accreditation 2019-2022) and the European Union (European Regional Development Fund-ERDF). D.G.-S. is financially supported by Xunta de Galicia, a postdoctoral contract ED481B/2018/091.

Institutional Review Board Statement: Not applicable.

Informed Consent Statement: Not applicable.

Data Availability Statement: The data presented in this study are available in NCBI GenBank under the BioSample accession number: SAMN15184427, BioProject: PRJNA638244. All scripts used are available at: https://bitbucket.org/MonikaTC/tunjic-cvitanic-et-al.-2021/src/master/ (added on 1 May 2021). 
Acknowledgments: We would like to thank Tanja Vojvoda Zeljko for her engagement in obtaining bivalve specimens, and Lucija Horvat for the help with the microscopy performed at Ruđer Bošković Institute.

Conflicts of Interest: The authors declare no conflict of interest. The funders had no role in the design of the study; in the collection, analyses, or interpretation of data; in the writing of the manuscript, or in the decision to publish the results.

\section{References}

1. Charlesworth, B.; Sniegowski, P.; Stephan, W. The Evolutionary Dynamics of Repetitive DNA in Eukaryotes. Nature 1994, 371, 215-220. [CrossRef] [PubMed]

2. Schmidt, T.; Heslop-Harrison, J.S. Genomes, Genes and Junk: The Large-Scale Organization of Plant Chromosomes. Trends Plant Sci. 1998, 3, 195-199. [CrossRef]

3. Plohl, M.; Luchetti, A.; Meštrović, N.; Mantovani, B. Satellite DNAs between Selfishness and Functionality: Structure, Genomics and Evolution of Tandem Repeats in Centromeric (Hetero) Chromatin. Gene 2008, 409, 72-82. [CrossRef]

4. Garrido-Ramos, M.A. Satellite DNA: An Evolving Topic. Genes 2017, 8, 230. [CrossRef] [PubMed]

5. Hartley, G.; O'Neill, R.J. Centromere Repeats: Hidden Gems of the Genome. Genes 2019, 10, 223. [CrossRef] [PubMed]

6. Tørresen, O.K.; Star, B.; Mier, P.; Andrade-Navarro, M.A.; Bateman, A.; Jarnot, P.; Gruca, A.; Grynberg, M.; Kajava, A.V.; Promponas, V.J.; et al. Tandem Repeats Lead to Sequence Assembly Errors and Impose Multi-Level Challenges for Genome and Protein Databases. Nucleic Acids Res. 2019, 47, 10994-11006. [CrossRef] [PubMed]

7. Šatović, E.; Tunjić Cvitanić, M.; Plohl, M. Tools and Databases for Solving Problems in Detection and Identification of Repetitive DNA Sequences. Period. Biol. 2020, 121-122, 7-14. [CrossRef]

8. Kim, Y.B.; Oh, J.H.; Mciver, L.J.; Rashkovetsky, E.; Michalak, K.; Garner, H.R.; Kang, L.; Nevo, E.; Korol, A.B.; Michalak, P. Divergence of Drosophila melanogaster Repeatomes in Response to a Sharp Microclimate Contrast in Evolution. Proc. Natl. Acad. Sci. USA 2014, 111, 10630-10635. [CrossRef]

9. Ruiz-Ruano, F.J.; López-León, M.D.; Cabrero, J.; Camacho, J.P.M. High-Throughput Analysis of the Satellitome Illuminates Satellite DNA Evolution. Sci. Rep. 2016, 6, 1-14. [CrossRef] [PubMed]

10. Palacios-Gimenez, O.M.; Koelman, J.; Flores, M.P.; Bradford, T.M.; Jones, K.K.; Cooper, S.J.B.; Kawakami, T.; Suh, A. Comparative Analysis of Morabine Grasshopper Genomes Reveals Highly Abundant Transposable Elements and Rapidly Proliferating Satellite DNA Repeats. BMC Biol. 2020, 18. [CrossRef]

11. Utsunomia, R.; de Silva, D.M.Z.; Ruiz-Ruano, F.J.; Goes, C.A.G.; Melo, S.; Ramos, L.P.; Oliveira, C.; Porto-Foresti, F.; Foresti, F.; Hashimoto, D.T. Satellitome Landscape Analysis of Megaleporinus macrocephalus (Teleostei, Anostomidae) Reveals Intense Accumulation of Satellite Sequences on the Heteromorphic Sex Chromosome. Sci. Rep. 2019, 9, 1-10. [CrossRef] [PubMed]

12. Sader, M.; Vaio, M.; Cauz-Santos, L.A.; Dornelas, M.C.; Vieira, M.L.C.; Melo, N.; Pedrosa-Harand, A. Large vs small genomes in Passiflora: The influence of the mobilome and the satellitome. Planta 2021, 253, 1-18. [CrossRef]

13. Macas, J.; Novak, P.; Pellicer, J.; Cizkova, J.; Koblizkova, A.; Neumann, P.; Fukova, I.; Dolezel, J.; Kelly, L.J.; Leitch, I.J. In Depth Characterization of Repetitive DNA in 23 Plant Genomes Reveals Sources of Genome Size Variation in the Legume Tribe Fabeae. PLoS ONE 2015, 10, e0143424. [CrossRef] [PubMed]

14. Klemme, S.; Banaei-Moghaddam, A.M.; Macas, J.; Wicker, T.; Novák, P.; Houben, A. High-Copy Sequences Reveal Distinct Evolution of the Rye B Chromosome. New Phytol. 2013, 199, 550-558. [CrossRef]

15. Palacios-Gimenez, O.M.; Dias, G.B.; De Lima, L.G.; Kuhn, G.C.E.S.; Ramos, É.; Martins, C.; Cabral-De-Mello, D.C. HighThroughput Analysis of the Satellitome Revealed Enormous Diversity of Satellite DNAs in the Neo-Y Chromosome of the Cricket Eneoptera surinamensis. Sci. Rep. 2017, 7, 1-11. [CrossRef] [PubMed]

16. Belyayev, A.; Josefiová, J.; Jandová, M.; Kalendar, R.; Krak, K.; Mandák, B. Natural History of a Satellite DNA Family: From the Ancestral Genome Component to Species-Specific Sequences, Concerted and Non-Concerted Evolution. Int. J. Mol. Sci. 2019, 20, 1201. [CrossRef] [PubMed]

17. Dias, G.B.; Heringer, P.; Svartman, M.; Kuhn, G.C.S.S. Helitrons Shaping the Genomic Architecture of Drosophila: Enrichment of DINE-TR1 in $\alpha$ - and $\beta$-Heterochromatin, Satellite DNA Emergence, and PiRNA Expression. Chromosome Res. 2015, 23, 597-613. [CrossRef]

18. Brajković, J.; Feliciello, I.; Bruvo-Mađarić, B.; Ugarković, D.W. Satellite DNA-like Elements Associated with Genes within Euchromatin of the Beetle Tribolium castaneum. G3 Genes Genomes Genet. 2012, 2, 931-941. [CrossRef]

19. Šatović, E.; Vojvoda Zeljko, T.; Luchetti, A.; Mantovani, B.; Plohl, M. Adjacent Sequences Disclose Potential for Intra-Genomic Dispersal of Satellite DNA Repeats and Suggest a Complex Network with Transposable Elements. BMC Genom. 2016, 17, 997. [CrossRef]

20. Feliciello, I.; Pezer, Ž.; Kordiš, D.; Mađarić, B.B.; Ugarković, Đ. Evolutionary History of Alpha Satellite DNA Repeats Dispersed within Human Genome Euchromatin. Genome Biol. Evol. 2020, 14561197. [CrossRef]

21. Vondrak, T.; Ávila Robledillo, L.; Novák, P.; Koblížková, A.; Neumann, P.; Macas, J. Characterization of repeat arrays in ultra-long nanopore reads reveals frequent origin of satellite DNA from retrotransposon-derived tandem repeats. Plant J. 2020, 101, 484-500. [CrossRef] 
22. Gaffney, P.M.; Pierce, J.C.; Mackinley, A.G.; Titchen, D.A.; Glenn, W.K. Pearl, a Novel Family of Putative Transposable Elements in Bivalve Mollusks. J. Mol. Evol. 2003, 56, 308-316. [CrossRef]

23. Dias, G.B.; Svartman, M.; Delprat, A.; Ruiz, A.; Kuhn, G.C.S.S. Tetris Is a Foldback Transposon That Provided the Building Blocks for an Emerging Satellite DNA of Drosophila virilis. Genome Biol. Evol. 2014, 6, 1302-1313. [CrossRef]

24. Luchetti, A. TerMITEs: Miniature Inverted-Repeat Transposable Elements (MITEs) in the Termite Genome (Blattodea: Termitoidae). Mol. Genet. Genomics 2015, 290, 1499-1509. [CrossRef]

25. Wijsman, J.W.M.; Fang, J.; Roncarati, A. Global Production of Marine Bivalves. Trends and Challenges. In Goods and Services of Marine Bivalves; Smaal, A.C., Ferreira, J.G., Grant, J., Petersen, J.K., Strand, Ø., Eds.; Springer International Publishing: Cham, Switzerland, 2018; pp. 7-26. [CrossRef]

26. Vaughn, C.C.; Hoellein, T.J. Bivalve Impacts in Freshwater and Marine Ecosystems. Annu. Rev. Ecol. Evol. Syst. 2018, 49, 183-208. [CrossRef]

27. Suárez-Ulloa, V.; Fernández-Tajes, J.; Manfrin, C.; Gerdol, M.; Venier, P.; Eirín-López, J.M. Bivalve Omics: State of the Art and Potential Applications for the Biomonitoring of Harmful Marine Compounds. Mar. Drugs 2013, 11, 4370-4389. [CrossRef] [PubMed]

28. Gomes-dos-Santos, A.; Lopes-Lima, M.; Castro, L.F.C.; Froufe, E. Molluscan Genomics: The Road so Far and the Way Forward. Hydrobiologia 2020, 847, 1705-1726. [CrossRef]

29. Fernández Robledo, J.A.; Yadavalli, R.; Allam, B.; Gerdol, M.; Greco, S.; Stevick, R.J.; Zhang, Y.; Heil, C.A.; Tracy, A.N.; Bishopbailey, D.; et al. From the Raw Bar to the Bench: Bivalves as Models for Human Health. Dev. Comp. Immunol. 2020, 92, 260-282. [CrossRef] [PubMed]

30. Murgarella, M.; Puiu, D.; Novoa, B.; Figueras, A.; Posada, D.; Canchaya, C. A First Insight into the Genome of the Filter- Feeder Mussel Mytilus galloprovincialis. PLoS ONE 2016, 11, e0151561. [CrossRef]

31. Zhang, G.; Fang, X.; Guo, X.; Li, L.; Luo, R.; Xu, F.; Yang, P.; Zhang, L.; Wang, X.; Qi, H.; et al. The Oyster Genome Reveals Stress Adaptation and Complexity of Shell Formation. Nature 2012, 490, 49-54. [CrossRef] [PubMed]

32. Takeuchi, T.; Kawashima, T.; Koyanagi, R.; Gyoja, F.; Tanaka, M.; Ikuta, T.; Shoguchi, E.; Fujiwara, M.; Shinzato, C.; Hisata, K.; et al. Draft Genome of the Pearl Oyster Pinctada fucata: A Platform for Understanding Bivalve Biology. DNA Res. 2012, 19, 117-130. [CrossRef]

33. Mun, S.; Kim, Y.J.; Markkandan, K.; Shin, W.; Oh, S.; Woo, J.; Yoo, J.; An, H.; Han, K. The Whole-Genome and Transcriptome of the Manila Clam (Ruditapes Philippinarum). Genome Biol. Evol. 2017, 9, 1487-1498. [CrossRef] [PubMed]

34. Šatović, E.; Vojvoda Zeljko, T.; Plohl, M. Characteristics and Evolution of Satellite DNA Sequences in Bivalve Mollusks. Eur. Zool. J. 2018, 85, 95-104. [CrossRef]

35. Tunjić Cvitanić, M.; Vojvoda Zeljko, T.; Pasantes, J.J.; García-Souto, D.; Gržan, T.; Despot-Slade, E.; Plohl, M.; Šatović, E. Sequence Composition Underlying Centromeric and Heterochromatic Genome Compartments of the Pacific Oyster Crassostrea gigas. Genes 2020, 11, 695. [CrossRef]

36. Clabby, C.; Goswami, U.; Flavin, F.; Wilkins, N.P.; Houghton, J.A.; Powell, R. Cloning, Characterization and Chromosomal Location of a Satellite DNA from the Pacific Oyster, Crassostrea gigas. Gene 1996, 168, 205-209. [CrossRef]

37. Vojvoda Zeljko, T.; Pavlek, M.; Meštrović, N.; Plohl, M. Satellite DNA-like Repeats Are Dispersed throughout the Genome of the Pacific Oyster Crassostrea gigas Carried by Helentron Non-Autonomous Mobile Elements. Sci. Rep. 2020, 10, 1-12. [CrossRef]

38. Thomas, J.; Pritham, E.J. Helitrons, the Eukaryotic Rolling-Circle Transposable Elements. Microbiol. Spectr. 2015, 3, 1-32. [CrossRef]

39. Peñaloza, C.; Gutierrez, A.P.; Eory, L.; Wang, S.; Guo, X.; Archibald, A.L.; Bean, T.P.; Houston, R.D. A Chromosome-Level Genome Assembly for the Pacific Oyster (Crassostrea gigas). GigaScience 2021, 10, 1-9. [CrossRef]

40. López-Flores, I.; de la Herrán, R.; Garrido-Ramos, M.A.; Boudry, P.; Ruiz-Rejón, C.; Ruiz-Rejón, M. The Molecular Phylogeny of Oysters Based on a Satellite DNA Related to Transposons. Gene 2004, 339, 181-188. [CrossRef]

41. Jurka, J.; Kapitonov, V.V.; Pavlicek, A.; Klonowski, P.; Kohany, O.; Walichiewicz, J. Repbase Update, a Database of Eukaryotic Repetitive Elements. Cytogenet. Genome Res. 2005, 110, 462-467. [CrossRef]

42. Yang, H.P.; Barbash, D.A. Abundant and Species-Specific DINE-1 Transposable Elements in 12 Drosophila Genomes. Genome Biol. 2008, 9, 1-14. [CrossRef]

43. Šatović, E.; Plohl, M. Tandem Repeat-Containing MITE Elements in the Clam Donax trunculus. Genome Biol. Evol. 2013, 5, 2549-2559. [CrossRef]

44. Xiong, W.; Dooner, H.K.; Du, C. Rolling-Circle Amplification of Centromeric Helitrons in Plant Genomes. Plant J. 2016, 88, 1038-1045. [CrossRef]

45. Wang, Y.; Xu, Z.; Guo, X. A Centromeric Satellite Sequence in the Pacific Oyster (Crassostrea gigas Thunberg) Identified by Fluorescence In Situ Hybridization. Mar. Biotechnol. 2001, 3, 486-492. [CrossRef] [PubMed]

46. Cross, I.; Díaz, E.; Sánchez, I.; Rebordinos, L. Molecular and Cytogenetic Characterization of Crassostrea angulata Chromosomes. Aquaculture 2005, 247, 135-144. [CrossRef]

47. Louzada, S.; Lopes, M.; Ferreira, D.; Adega, F.; Escudeiro, A.; Gama-carvalho, M.; Chaves, R. Decoding the role satellite DNA in genome Architecture and Plasticity—An Evolutionary and Clinical Affair. Genes 2020, 11, 72. [CrossRef] [PubMed]

48. Bouilly, K.; Chaves, R.; Leitao, A.; Benabdelmouna, A.; Guedes-Pinto, H. Chromosomal Organization of Simple Sequence Repeats in Chromosome Patterns. J. Genet. 2008, 87, 119-125. [CrossRef] 
49. Van Dijk, E.L.; Jaszczyszyn, Y.; Naquin, D.; Thermes, C. The Third Revolution in Sequencing Technology. Trends Genet. 2018, 34, 666-681. [CrossRef] [PubMed]

50. Sedlazeck, F.J.; Lee, H.; Darby, C.A.; Schatz, M.C. Piercing the Dark Matter: Bioinformatics of Long-Range Sequencing and Mapping. Nat. Rev. Genet. 2018, 19, 329-346. [CrossRef]

51. Wang, X.; Xu, W.; Wei, L.; Zhu, C.; He, C.; Song, H.; Cai, Z.; Yu, W.; Jiang, Q.; Li, L.; et al. Nanopore Sequencing and De Novo Assembly of a Black-Shelled Pacific Oyster (Crassostrea gigas) Genome. Front. Genet. 2019, 10, 1211. [CrossRef]

52. Pita, S.; Panzera, F.; Mora, P.; Vela, J.; Cuadrado, Á.; Sánchez, A.; Palomeque, T.; Lorite, P. Comparative Repeatome Analysis on Triatoma infestans Andean and Non-Andean Lineages, Main Vector of Chagas Disease. PLoS ONE 2017, 12, e0181635. [CrossRef] [PubMed]

53. Boštjančić, L.L.; Bonassin, L.; Anušić, L.; Lovrenčić, L.; Besendorfer, V.; Maguire, I.; Grandjean, F.; Austin, C.M.; Greve, C.; Hamadou, A.B.; et al. The Pontastacus leptodactylus (Astacidae) Repeatome Provides Insight Into Genome Evolution and Reveals Remarkable Diversity of Satellite DNA. Front. Genet. 2021, 11, 611745. [CrossRef] [PubMed]

54. Heslop-Harrison, J.S.P.; Schwarzacher, T. Nucleosomes and Centromeric DNA Packaging. Proc. Natl. Acad. Sci. USA 2013, 110, 19974-19975. [CrossRef]

55. Meštrović, N.; Mravinac, B.; Pavlek, M.; Vojvoda-Zeljko, T.; Šatović, E.; Plohl, M. Structural and Functional Liaisons between Transposable Elements and Satellite DNAs. Chromosome Res. 2015, 23, 583-596. [CrossRef]

56. Alkan, C.; Ventura, M.; Archidiacono, N.; Rocchi, M.; Sahinalp, S.C.; Eichler, E.E. Organization and Evolution of Primate Centromeric DNA from Whole-Genome Shotgun Sequence Data. PLoS Comput. Biol. 2007, 3, 1807-1818. [CrossRef]

57. Scalvenzi, T.; Pollet, N. Insights on Genome Size Evolution from a Miniature Inverted Repeat Transposon Driving a Satellite DNA. Mol. Phylogenet. Evol. 2014, 81, 1-9. [CrossRef] [PubMed]

58. Paço, A.; Freitas, R.; Vieira-Da-Silva, A. Conversion of DNA Sequences: From a Transposable Element to a Tandem Repeat or to a Gene. Genes 2019, 10, 1014. [CrossRef]

59. Hikosaka, A.; Kawahara, A. Lineage-Specific Tandem Repeats Riding on a Transposable Element of MITE in Xenopus Evolution: A New Mechanism for Creating Simple Sequence Repeats. J. Mol. Evol. 2004, 59, 738-746. [CrossRef] [PubMed]

60. Izsvák, Z.; Ivics, Z.; Shimoda, N.; Mohn, D.; Okamoto, H.; Hackett, P.B. Short Inverted-Repeat Transposable Elements in Teleost Fish and Implications for a Mechanism of Their Amplification. J. Mol. Evol. 1999, 48, 13-21. [CrossRef] [PubMed]

61. Miller, W.J.; Nagel, A.; Bachmann, J.; Bachmann, L. Evolutionary Dynamics of the SGM Transposon Family in the Drosophila obscura Species Group. Mol. Biol. Evol. 2000, 17, 1597-1609. [CrossRef]

62. Kuhn, G.C.S.; Heslop-Harrison, J.S. Characterization and Genomic Organization of PERI, a Repetitive DNA in the Drosophila buzzatii Cluster Related to DINE-1 Transposable Elements and Highly Abundant in the Sex Chromosomes. Cytogenet. Genome Res. 2011, 132, 79-88. [CrossRef] [PubMed]

63. Thomas, J.; Vadnagara, K.; Pritham, E.J. DINE-1, the Highest Copy Number Repeats in Drosophila Melanogaster Are NonAutonomous Endonuclease-Encoding Rolling-Circle Transposable Elements (Helentrons). Mob. DNA 2014, 5, 18. [CrossRef] [PubMed]

64. Novák, P.; Robledillo, L.Á.; Koblížková, A.; Vrbová, I.; Neumann, P.; Macas, J. TAREAN: A Computational Tool for Identification and Characterization of Satellite DNA from Unassembled Short Reads. Nucleic Acids Res. 2017, 45, e111. [CrossRef] [PubMed]

65. Novák, P.; Neumann, P.; Pech, J.; Steinhaisl, J.; Macas, J. RepeatExplorer: A Galaxy-Based Web Server for Genome-Wide Characterization of Eukaryotic Repetitive Elements from Next-Generation Sequence Reads. Bioinformatics 2013, 29 , 792-793. [CrossRef] [PubMed]

66. Martínez-Expósito, M.J.; Pasantes, J.J.; Méndez, J. NOR Activity in Larval and Juvenile Mussels (Mytilus galloprovincialis Lmk.). J. Exp. Mar. Bio. Ecol. 1994, 175, 155-165. [CrossRef]

67. Pérez-García, C.; Morán, P.; Pasantes, J.J. Cytogenetic Characterization of the Invasive Mussel Species Xenostrobus securis Lmk. (Bivalvia: Mytilidae). Genome 2011, 54, 771-778. [CrossRef] 